\title{
A Thin Lens Model for Charged-Particle RF Accelerating Gaps
}

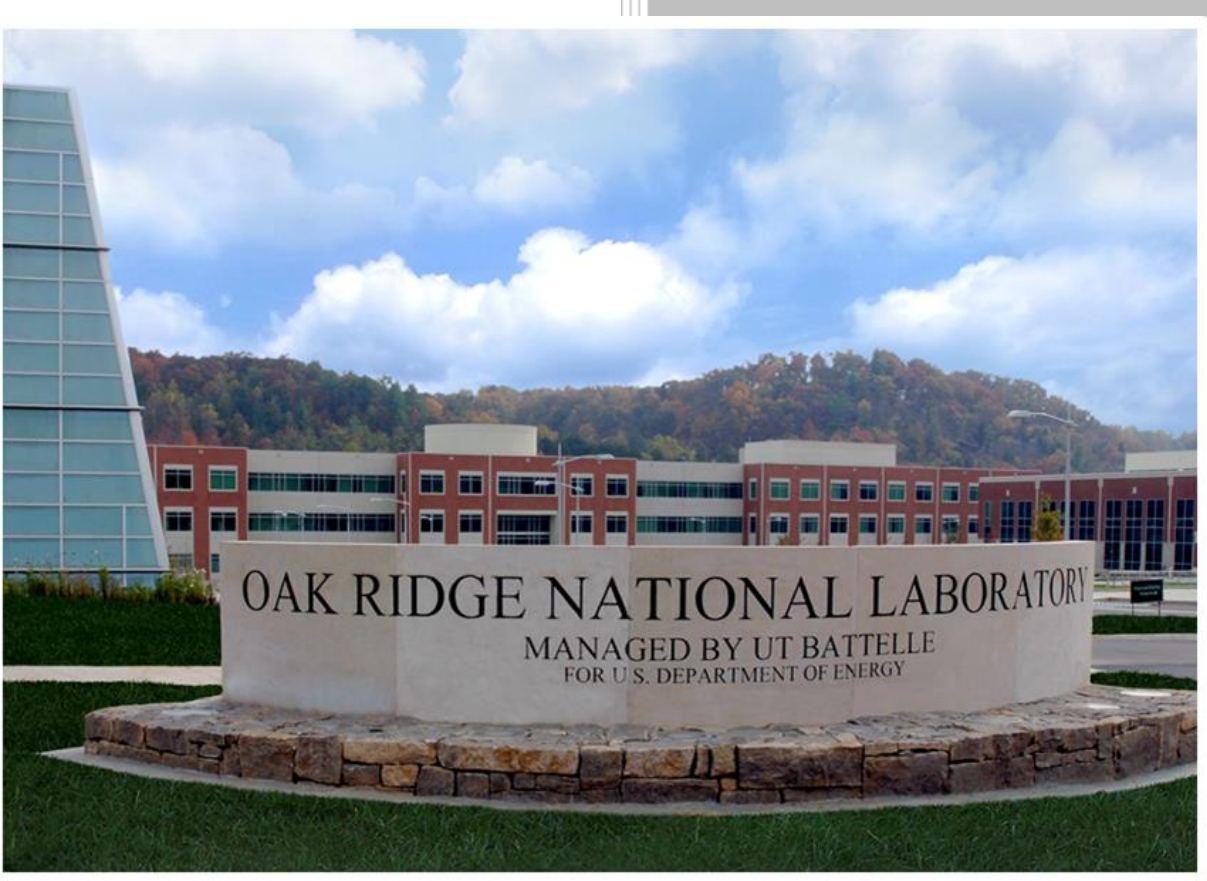

Approved for public release.

Distribution is unlimited.

Christopher K. Allen July 27, 2017 


\section{DOCUMENT AVAILABILITY}

Reports produced after January 1, 1996, are generally available free via US Department of Energy (DOE) SciTech Connect.

Website http://www.osti.gov/scitech/

Reports produced before January 1, 1996, may be purchased by members of the public from the following source:

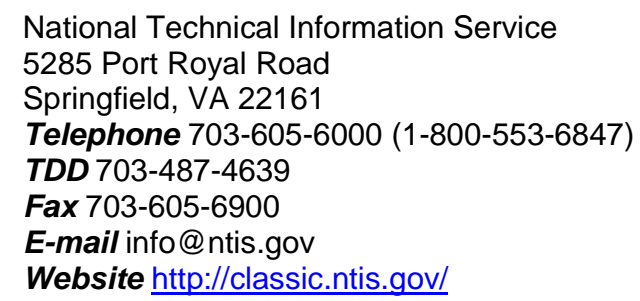

Reports are available to DOE employees, DOE contractors, Energy Technology Data Exchange representatives, and International Nuclear Information System representatives from the following source:

Office of Scientific and Technical Information

PO Box 62

Oak Ridge, TN 37831

Telephone 865-576-8401

Fax 865-576-5728

E-mail reports@osti.gov

Website http://www.osti.gov/contact.html

This report was prepared as an account of work sponsored by an agency of the United States Government. Neither the United States Government nor any agency thereof, nor any of their employees, makes any warranty, express or implied, or assumes any legal liability or responsibility for the accuracy, completeness, or usefulness of any information, apparatus, product, or process disclosed, or represents that its use would not infringe privately owned rights. Reference herein to any specific commercial product, process, or service by trade name, trademark, manufacturer, or otherwise, does not necessarily constitute or imply its endorsement, recommendation, or favoring by the United States Government or any agency thereof. The views and opinions of authors expressed herein do not necessarily state or reflect those of the United States Government or any agency thereof. 
Research Accelerator Division

\title{
A Thin Lens Model for Charge-Particle RF Accelerating Gaps
}

\author{
Christopher K. Allen
}

Date Published: July 27, 2017

\author{
Prepared by \\ OAK RIDGE NATIONAL LABORATORY \\ Oak Ridge, TN 37831-6283 \\ managed by \\ UT-BATTELLE, LLC \\ for the \\ US DEPARTMENT OF ENERGY \\ under contract DE-AC05-00OR22725
}





\section{CONTENTS}

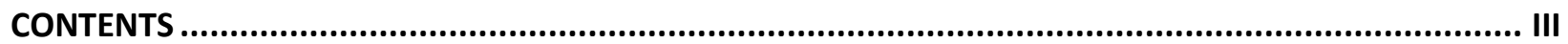

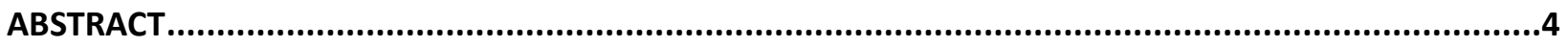

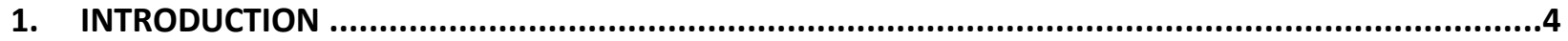

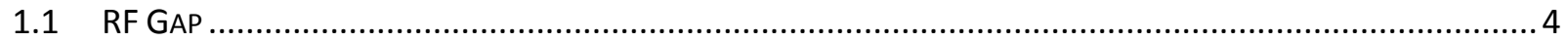

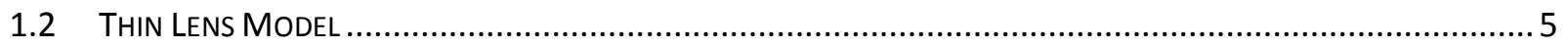

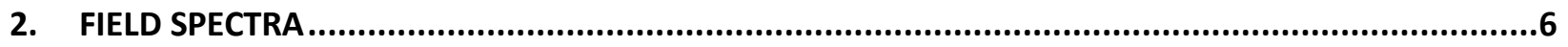

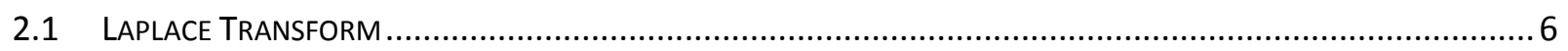

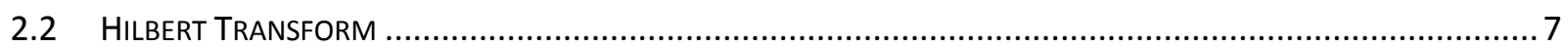

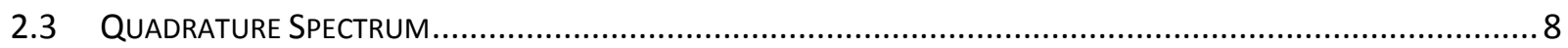

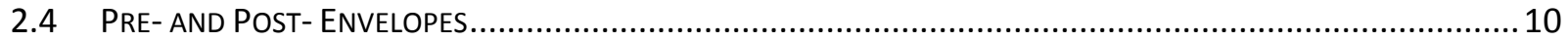

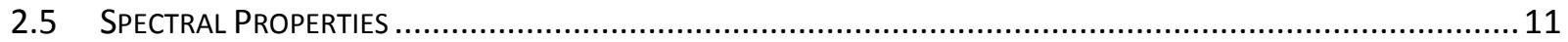

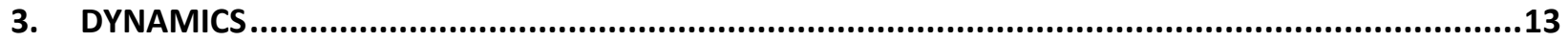

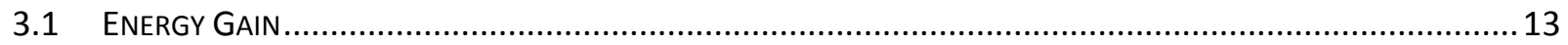

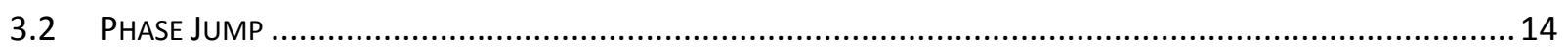

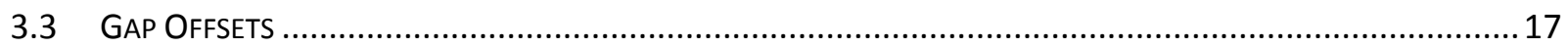

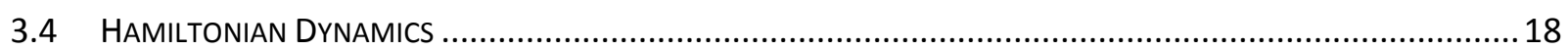

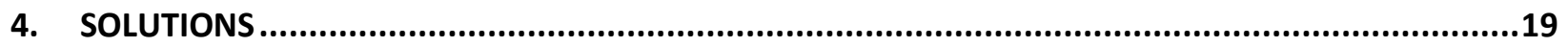

4.1 ALGORITHM 1: MID-GAP WAVE NUMBER

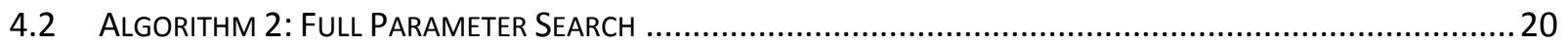

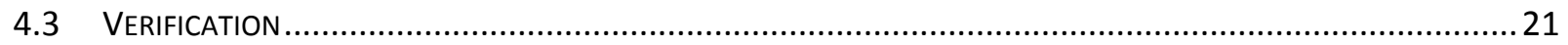

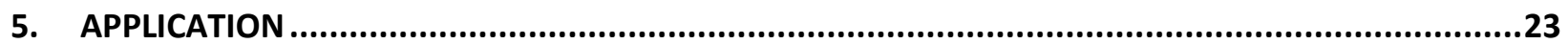

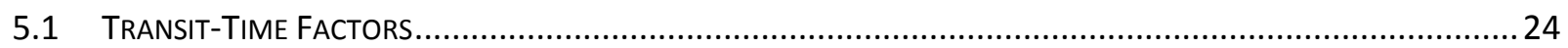

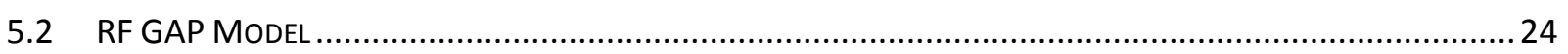

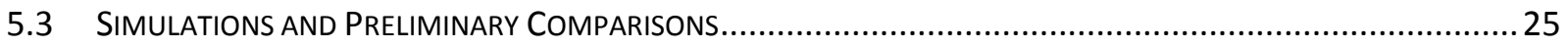

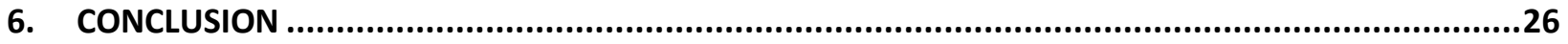

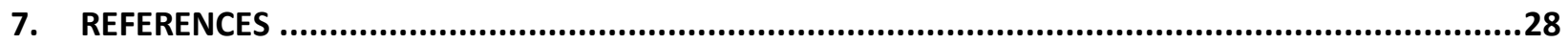




\begin{abstract}
Presented is a thin-lens model for an RF accelerating gap that considers general axial fields and is accurate in the case where energy gain within the gap is small with respect to initial particle energy. Both the cosine and sine transit time factors (i.e., Fourier transforms) are required plus two additional functions; the Hilbert transforms the transit-time factors. The combination yields a complex-valued Hamiltonian rotating in the complex plane with synchronous phase. Using Hamiltonians the phase and energy gains are computed independently in the pre-gap and post-gap regions then aligned using the asymptotic values of wave number. Derivations of these results are outlined, examples are shown, and simulations with the model are presented.
\end{abstract}

\title{
1. INTRODUCTION
}

The thin-lens model for a radio-frequency (RF) accelerating gap has been well developed by Lapostolle [1], Weiss [2], Wangler [3] and is widely used in practice (for example, see [4]). Shishlo and Holmes develop general simulation models for charged-particle acceleration using RF gaps starting from first principles and the field equations [5]; they provide a concise compendium of formulae and techniques including the thin-lens model. The current work, however, is restrictive and focuses exclusively on the thin-lens RF gap model.

Although the model of a thin-lens RF gap is straightforward, the required analysis needed to produce the operational results can be somewhat involved. Here the model is analysed using the tools of system theory and signal processing in order to provide a coherent framework and yielding a Hamiltonian-like dynamics that might allow for further generalization. Also, some amount of mathematical manipulation is avoided by using tools from analysis such as the Laplace and Hilbert transforms, in particular with respect to their convolution and differentiation properties.

The current paper is a companion to one previously published within the IPAC 2017 conference proceedings [6]. Due to space limitations only a portion of this work could be presented, and little of the derivations. The purpose here is to further the description of the thin-lens model and to obviate the methods used to generate the results of the original paper.

\subsection{RF GAP}

Referring to Figure 1, one sees a cylindrical coordinate system where axial position is denoted $z$ and the radial position $r$. The figure depicts the geometry of an idealized RF cell, or $R F$ gap, within a cylindrically symmetric RF accelerating cavity. Although there are many types of accelerating structures and, consequently, gap geometries, the gap geometry pictured in Figure 1 is general enough to cover most practical situations. Take the gap center position to be $z=0$ and the gap length to be $L$ where the value $L$ usually has some physical significance in the gap design. The cell "starts" at $z=-L / 2$ and "ends" at $z=+L / 2$. The cell aperture radius is denoted $a$, but it is not explicitly needed in the sequel. The structure is rotationally

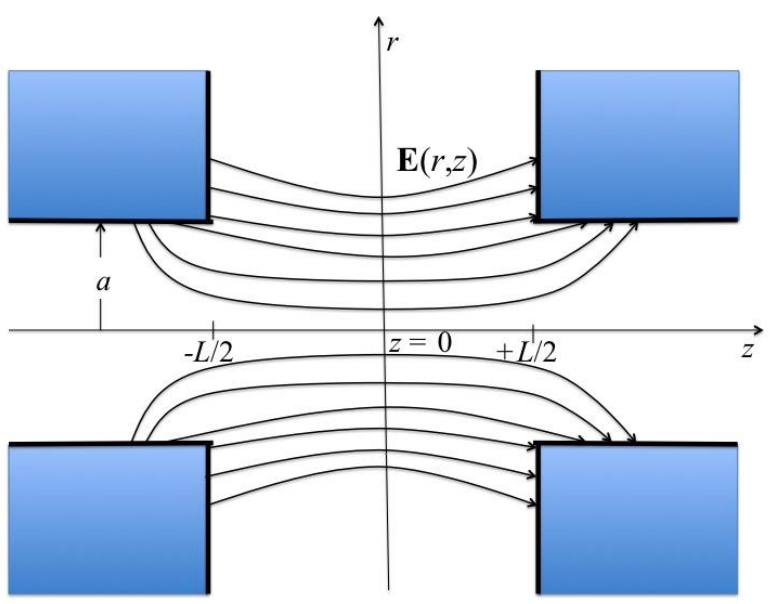

Figure 1: RF gap geometry symmetric about the longitudinal $z$-axis. The $z$-axis is also the axis of particle propagation. That is, the 
charged particle beam is designed to propagation through the RF cavity along the $z$-axis. As indicated in Figure 1 the region around $z=0$ has longitudinal electric fields that are assumed to be strong, especially on the $z$-axis. The RF cavity is designed so in order to provide acceleration by these electric fields in the direction of propagation. On either side of the strong field region, $z<-L / 2$ and $z>+L / 2$, the fields are relatively small and insignificant to the dynamics. Typically these regions are modeled as drifts while the strong field region would be considered the actual RF gap. In fact, in the thin-lens model the entire strong field region $z \in\left[-\frac{L}{2},+\frac{L}{2}\right]$ is replaced by a zero-length element at $z=0$ where the action of the fields is applied instantaneously, while the regions $z<0$ and $z>0$ are then modeled as drift spaces.

The RF gaps are located in some type of resonant RF cavity which is intended to be operated at a given frequency $f$. Thus, it is assumed that the RF fields are time harmonic and that the electric field vector $\mathbb{E}$ has the form

$$
\mathfrak{E}(r, z ; t)=\mathbf{E}(r, z) \cos \left(\omega t+\phi_{0}\right),
$$

where $\mathbf{E}(r, z)$ is the (independent) spatial variation of the fields, $\omega=2 \pi f$ is the RF angular frequency, and $\phi_{0}$ is the RF phase at time $t=0$. The spatial dependence of $\mathbf{E}(r, z)$ is determined by the cavity geometry and is the focus of cavity design. In order that there be strong accelerating electric field the RF cavity operates in some transverse magnetic mode, typically the fundamental. Thus all field vector components $\mathbf{E}(r, z)$ can be expanded from the axial electric field component $E_{z}(r, z)$. From Maxwell's equations the on-axis longitudinal field $E_{z}(0, z)$ contains all the information of the electromagnetic fields in the region of the beam axis (i.e., along the design trajectory). And so by Fourier's theorem, the spectrum of $E_{z}(0, z)$ contains all the information of corresponding design fields. Consequently the radial dependence of the longitudinal field component $E_{z}$ is usually ignored and the shorthand notation $E_{z}(z)$ is used.

\subsection{THIN LENS MODEL}

In the thin-lens model for an RF accelerating gap, particle energy $W$ is constant upstream

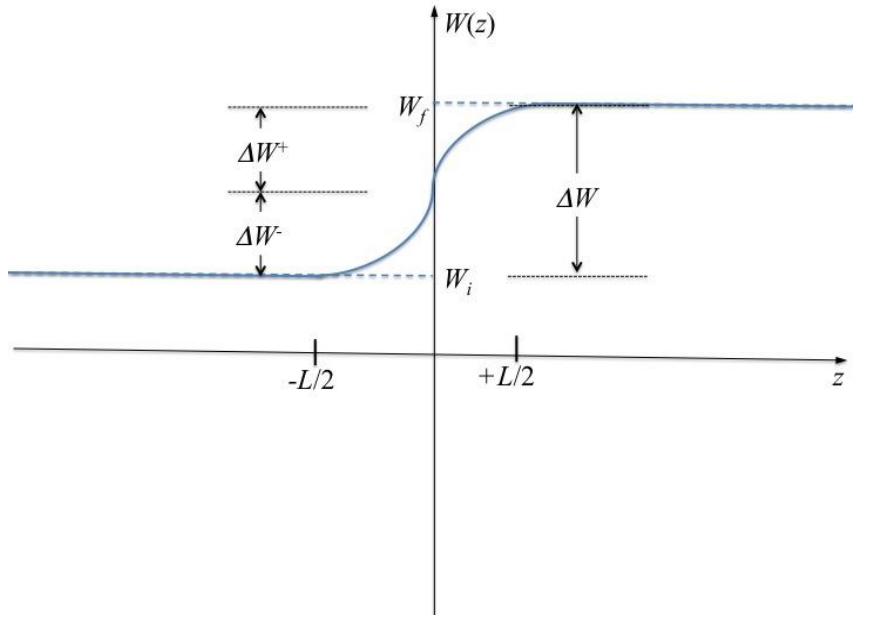

Figure 2: energy gain through gap and downstream of the gap but experiences an impulsive energy gain $\Delta W$ at the center $z=0$. (Thus, the particle wave number $k$ is also constant before and after the gap, differing by a value $\Delta k$ consistent with $\Delta W)$. This situation is depicted in Figure 2. In order to satisfy Hamiltonian dynamics the particle phase $\phi$, being the conjugate coordinate to $W$, must also experience an impulse $\Delta \phi$ at the gap center; this situation is shown in Figure 4. In the thin-lens model, the phase advance $\phi(z)$ is linear on either side of the gap due to the constant energy and, hence, constant wave number $k$. From Figure 4 one sees that the phase jump $\Delta \phi$ is necessary to "re-align" the linear phase advance $\phi(z)$ of the thin-lens model on either side of the gap.

Given this model for the accelerating RF gap, the objective is to determine the correct pair $(\Delta \phi, \Delta W)$ for an RF gap given its longitudinal field profile $\mathfrak{E}_{z}(z ; t)$ and the initial particle coordinates $\left(\phi_{0}^{-}, W^{-}\right)$. Assuming that the longitudinal field component $\mathfrak{E}_{z}$ is harmonic, the spatial and time dependence can be separated as

$$
\mathfrak{E}_{z}(z ; t)=E_{z}(z) \cos \phi(t)
$$


where $E_{z}(\cdot)$ is the axial profile (i.e., $r=0$ ) of the longitudinal electric field component. The phase of the $\mathrm{RF}$ is given by

$$
\phi(t)=\omega t+\phi_{0},
$$

where $\omega=2 \pi f$ is the RF angular frequency and $\phi_{0}$ is the RF phase at (arbitrary) time $t=0$. Noting that $\phi(t)$ is the RF phase seen by the particle at time $t$, we can express $\phi$ as a function of particle position $z$ by invoking its positional dependence $z(t)$. Expressing particle velocity $\beta c$ as a function of position $z$,

$$
\begin{aligned}
\phi(z) & =2 \pi f \int_{0}^{z} \frac{1}{\beta(s) c} d s+\phi_{0}, \\
& =\int_{0}^{z} k(s) d s+\phi_{0}, \\
& \approx \bar{k} z+\phi_{0},
\end{aligned}
$$

where $\beta(z)$ is the normalized particle velocity with respect to the speed of light $c, k(z)=2 \pi / \beta(z) \lambda$ is the particle wave number at position $z, \lambda$ is the RF wavelength, and $\phi_{0}$ is again the RF phase as the particle crosses the gap center. Setting the limits of integration from 0 to $z$ requires that the particle cross the gap center $z=0$ at $t=0$. In the third line we have applied the approximation that the particle velocity is constant and equal to an averaged velocity $\bar{\beta}$ thus producing an averaged wave number $\bar{k}$. This approximation is probably the most presumptuous feature of the thin-lens model. It is accurate when the energy gain $\Delta W$ is small compared to the initial kinetic energy $W^{-}$of the particle, or whenever the phase advance $\Delta \phi$ is small within the gap. This approximation is generally accurate for hadron machines. It is addressed more fully in [7].

\section{FIELD SPECTRA}

The thin lens model for the RF gap is typically expressed in terms of the Panosfky equation [3], which contains an efficiency factor known as the transit time factor. There are various interpretations of the transit time factor but the essential feature is its spectral properties. Here we focus on the spectral properties of the axial field profile and express the spectrum in terms of the Laplace transform.

The analysis of Section 3 requires that the axial electric field be isolated on each side of the gap in order to compute phase jump and energy gain independently in the upstream and downstream regions. In signal processing applications the Hilbert transform is used to rotate a signal's spectrum in the complex plane. Using specific filter configurations the resulting spectrum can be isolated to positive frequencies or negative frequencies by forming the so-called pre-and post-envelopes. The same idea is applied here, but to the field spectrum, in order to isolate the axial fields on either side of the gap. In Section 3 we return to the physical analysis of the thin-lens RF gap using the results of this section.

\subsection{LAPLACE TRANSFORM}

The two-sided Laplace transform $\mathcal{L}_{2}$ of the real function $f$ is defined [8]

$$
\mathcal{L}_{2}[f(z)](\sigma) \triangleq A C \int_{-\infty}^{+\infty} f(z) e^{-\sigma z} d z,
$$

where $A C$ indicates analytic continuation and $\sigma=\mu+i k \in \mathbb{C}$ is the complex transform variable. The Laplace transform of $f$ exists so long as the above integral exists; the integral is referred to as the Laplace integral and denote $L_{2}[f]$. The (two-sided) inverse Laplace transform $\mathcal{L}_{2}^{-1}$ of the complex function $F$ is defined [8] 


$$
\mathcal{L}_{2}^{-1}[F(\sigma)](z)=\frac{1}{2 \pi} \int_{B r} F(\sigma) e^{\sigma z} d \sigma,
$$

where the integral, also known as the Bromwich integral, is taken over a curve $B r \subset \mathbb{C}$ of the form $B r=$ $\{\sigma \in \mathbb{C} \mid \operatorname{Re} \sigma=$ const. $\}$, that is, an infinite vertical line in the complex plane. The specific vertical line depends upon the convergence properties of the Laplace integral and is covered more in Subsection 2.5. (Suffice it to say that the convergence of the inverse transform depends upon the convergence of the forward transform and that the Bromwich contour usually lies in a vertical strip containing the origin.)

Denote by $\varepsilon_{z}$ the Laplace transform of the axial field $E_{z}$, that is,

$$
\mathcal{E}_{z}(\sigma) \triangleq \mathcal{L}_{2}\left[E_{z}(z)\right](\sigma) .
$$

(The existence of this transform is deferred until Subsection 2.5, Property 3.) Then for $\sigma=i k$ and applying the Euler identity

$$
\begin{aligned}
\mathcal{E}_{z}(i k) & =\int_{-\infty}^{+\infty} E_{z}(z) e^{-i k z} d z \\
& =\int_{-\infty}^{+\infty} E_{z}(z)(\cos k z-i \sin k z) d z \\
& =V_{0} T_{z}(k)-i V_{0} S_{z}(k),
\end{aligned}
$$

where

$$
V_{0} \triangleq \int_{-\infty}^{+\infty} E_{z}(z) d z
$$

is the potential across the gap, and the functions $T_{z}(\cdot)$ and $S_{z}(\cdot)$ are the cosine and sine Fourier transform components of $E_{z}(\cdot)$ defined as [9][10]

$$
\begin{aligned}
& T_{z}(k) \triangleq \frac{1}{V_{0}} \int_{-\infty}^{+\infty} E_{z}(z) \cos k z d z, \\
& S_{z}(k) \triangleq \frac{1}{V_{0}} \int_{-\infty}^{+\infty} E_{z}(z) \sin k z d z
\end{aligned}
$$

The transforms $T_{Z}(\cdot)$ and $S_{z}(\cdot)$ are referred to as the gap transit time factors in the beam physics literature. Thus, the Laplace transform $\varepsilon_{z}$ of the electric field profile evaluates to the transit-time factors along the imaginary axis $\sigma=i k$.

\subsection{HILBERT TRANSFORM}

The Hilbert transform $\mathcal{H}$ of real function $f$ is defined [11]

$$
\mathcal{H}[f](z) \triangleq P V \frac{1}{\pi} \int_{-\infty}^{+\infty} \frac{f(\zeta)}{z-\zeta} d \zeta=\frac{1}{\pi z} * f(z),
$$

where $P V$ is the Cauchy principle value. It is useful for spectrum shifting in signal processing [12]. In our case it is used for shifting the axial field profile itself in order to isolate the pre- and post-gap regions. It can be shown that the Hilbert transform is anti-reflexive, that is, $\mathcal{H} \circ \mathcal{H}=-1$ [11]. 
From Def. (11) the Hilbert transform is recognized as the convolution of $f(z)$ with the kernel $1 / \pi z$, denoted $f(z) * 1 / \pi z$. Thus, it is convenient to exploit the convolution properties of the Laplace transform when expressing the Hilbert transform; this is done through the signum function sgn $(\cdot)$. The signum function is defined

$$
\operatorname{sgn}(z) \triangleq\left\{\begin{array}{rl}
-1 & z<0 \\
0 & z=0 \\
+1 & z>0
\end{array}\right.
$$

The two-sided Laplace transform of $\operatorname{sgn}(z)$ does not exist in the strict sense, however, we can define a limiting form analogous to that done for the Fourier transform. Begin by defining the following exponential expression:

$$
\operatorname{sgn}_{e}(z, \epsilon) \triangleq\left\{\begin{array}{cc}
-e^{+\epsilon z} & z<0, \\
0 & z=0 \\
+e^{-\epsilon z} & z>0,
\end{array}\right.
$$

where we take $\epsilon>0$ so that $\operatorname{sgn}(z)=\lim _{\epsilon \searrow 0^{+}} \operatorname{sgn}_{e}(z, \epsilon)$ where the limit is taken from above (from the right). The Laplace integral of $\operatorname{sgn}_{e}(z, \epsilon)$ is

$$
L_{2}\left[\operatorname{sgn}_{e}(z, \epsilon)\right](\sigma)=-\int_{-\infty}^{0} e^{-(\sigma-\epsilon) z} d z+\int_{0}^{+\infty} e^{-(\sigma+\epsilon) z} d z,
$$

where the region of convergence for the first integral is the half plane $\operatorname{Re} \sigma<+\epsilon$ and the second integral converges for $\operatorname{Re} \sigma>-\epsilon$. So for complex $\sigma$ on the strip $-\epsilon<\operatorname{Re} \sigma<+\epsilon$ the Laplace integral $L_{2}\left[\operatorname{sgn}_{e}(z, \epsilon)\right]$ exists for all $\epsilon>0$ and, consequently, the Laplace transform exists and is

$$
\mathcal{L}_{2}\left[\operatorname{sgn}_{e}(z, \epsilon)\right](\sigma)=A C \frac{1}{\sigma-\epsilon}+\frac{1}{\sigma+\epsilon} .
$$

The Laplace transform of $\operatorname{sgn}(z)$, denote by $\mathcal{S}$, is found by taking the limit as $\epsilon \searrow 0^{+}$. The result is

$$
\begin{aligned}
\mathcal{S}(\sigma) & \triangleq \lim _{\epsilon \searrow 0^{+}} \mathcal{L}_{2}\left[\operatorname{sgn}_{e}(z, \epsilon)\right](\sigma) \\
& =\frac{2}{\sigma} .
\end{aligned}
$$

Since $\epsilon$ tends to zero from the right, the convergence strip $-\epsilon<\operatorname{Re} \sigma<+\epsilon$ is not empty but contains the imaginary axis $\operatorname{Re} \sigma=0$. As a Laplace transform, however, $\mathcal{S}(\sigma)$ is defined on the entire complex plane through analytic continuation (except for pole location $\sigma=0$ ). As far as inverse transforms are concerned however, the only available Bromwich contour is the imaginary axis.

\subsection{QUADRATURE SPECTRUM}

Define the electric field quadrature transform $\mathcal{E}_{q}$ as

$$
\mathcal{E}_{q}(\sigma) \triangleq \mathcal{L}_{2}\left[\operatorname{sgn}(\mathrm{z}) E_{z}(z)\right](\sigma) .
$$

It is helpful to define an abstract "quadrature field profile" $E_{q}(\cdot)$

$$
E_{q}(z) \triangleq \operatorname{sgn}(z) E_{z}(z),
$$

so that the quadrature spectra $\mathcal{E}_{q}$ is the transform of the quadrature field $E_{q}$, that is,

$$
\mathcal{E}_{q}(\sigma)=\mathcal{L}_{2}\left[E_{q}(z)\right](\sigma) .
$$

Then from the convolution theorem of the Laplace transform one obtains 


$$
\mathcal{E}_{q}(\sigma)=\mathcal{L}_{2}\left[\operatorname{sgn}(z) E_{z}(z)\right](\sigma)=\left(\mathcal{S} * \mathcal{E}_{z}\right)(\sigma),
$$

where $\mathcal{S} * \mathcal{E}_{z}$ indicates the complex convolution of complex functions $\mathcal{S}(\sigma)$ and $\mathcal{E}_{z}(\sigma)$. Following LePage [8], the complex convolution can be evaluated as either of the complex integrals

$$
\begin{aligned}
\mathcal{E}_{q}(\sigma) & =\frac{1}{2 \pi i} \int_{B r(\mathcal{S})} \mathcal{S}(s) \mathcal{E}_{z}(\sigma-s) d s, \\
& =\frac{1}{2 \pi i} \int_{B r\left(\mathcal{E}_{z}\right)} \mathcal{S}(\sigma-s) \mathcal{E}_{z}(s) d s,
\end{aligned}
$$

where $\operatorname{Br}(\mathcal{S})$ is the Bromwich contour for the signum transform $\mathcal{S}$, and $\operatorname{Br}\left(\mathcal{E}_{z}\right)$ is a Bromwich contour for the field transform $\varepsilon_{z}$. Recall that there is only one contour for $\mathcal{S}$, the imaginary axis. Bromwich contours for $\varepsilon_{z}$ lie in a contiguous vertical strip containing the origin (see Subsection 2.5). Evaluating $\mathcal{E}_{q}(\sigma)$ using the second expression yields

$$
\mathcal{E}_{q}(\sigma)=\frac{1}{\pi i} \int_{B r\left(\mathcal{E}_{z}\right)} \frac{1}{\sigma-s} \mathcal{E}_{z}(s) d s,
$$

Unfortunately we cannot blindly employ the residue theorem because, in general, $\varepsilon_{z}$ will have an essential singularity at infinity. Even so it is interesting to observe the result. The task is somewhat complicated since the contour $\operatorname{Br}\left(\mathcal{E}_{z}\right)$ can lie on either side of the pole at $s=\sigma$. Invoking the residue theorem without justification produces

$$
\mathcal{E}_{q}(\sigma)= \begin{cases}+2 \mathcal{E}_{z}(\sigma) & \operatorname{Re} \sigma<0, \\ -i \mathcal{H}\left[\mathcal{E}_{z}(\sigma)\right] & \operatorname{Re} \sigma=0, \\ -2 \mathcal{E}_{z}(\sigma) & \operatorname{Re} \sigma>0,\end{cases}
$$

where the signs follow from the pole location relative to the integration contour. In the above we see that the spectrum of $E_{Z}(z)$ is being rotated 180 degrees clockwise as the pole passes through the imaginary axis. The case of $\operatorname{Re} \sigma=0$ is special in that the contour intersects the pole and the resulting integral becomes the Hilbert transform. This is also the case where we can guarantee existence by Property 1 of Subsection 2.5.

Now consider the quadrature transit-time factors $T_{q}$ and $S_{q}$ for field $E_{q}(\cdot)$ defined analogously to Eqs. (10),

$$
\begin{aligned}
& T_{q}(k) \triangleq \frac{1}{V_{0}} \int_{-\infty}^{+\infty} E_{q}(z) \cos k z d z=\frac{1}{V_{0}} \int_{-\infty}^{+\infty} \operatorname{sgn}(z) E_{z}(z) \cos k z d z, \\
& S_{q}(k) \triangleq \frac{1}{V_{0}} \int_{-\infty}^{+\infty} E_{q}(z) \sin k z d z=\frac{1}{V_{0}} \int_{-\infty}^{+\infty} \operatorname{sgn}(z) E_{z}(z) \sin k z d z
\end{aligned}
$$

so that

$$
\mathcal{E}_{q}(i k)=V_{0} T_{q}(k)-i V_{0} S_{q}(k) .
$$

By the convolution property of the Laplace transform [8]

$$
\mathcal{H}\left[\mathcal{E}_{z}(i k)\right]=\frac{1}{i \pi k} * \mathcal{E}_{z}(i k)=i \mathcal{L}_{2}\left[\operatorname{sgn}(z) E_{z}(z)\right]=i \mathcal{L}_{2}\left[E_{q}(z)\right],
$$


which is the equivalent of Eqs. (23) for the case $\operatorname{Re} \sigma=0$, or more specifically $\sigma=i k$. From linearity of $\mathcal{H}$, Eqs. (26), Defs. (17) and (24)

$$
\begin{aligned}
& S_{q}(k)=+\mathcal{H}\left[T_{z}(k)\right], \\
& T_{q}(k)=-\mathcal{H}\left[S_{z}(k)\right] .
\end{aligned}
$$

By the anti-reflexivity property $\mathcal{H} \circ \mathcal{H}=-1$ the transit-time factors are recovered via

$$
\begin{aligned}
T_{Z}(k) & =-\mathcal{H}\left[S_{q}(k)\right], \\
S_{z}(k) & =+\mathcal{H}\left[T_{q}(k)\right] .
\end{aligned}
$$

In principle then $T_{q}$ and $S_{q}$ contain no new information and may be computed from $T_{z}$ and $S_{z}$. However, numerical Hilbert transforms are nontrivial and it may be more practical to compute the quadrature components directly [12].

\subsection{PRE- AND POST- ENVELOPES}

Here we define the spectral pre-envelope $\mathcal{E}^{-}$and post-envelope $\mathcal{E}^{+}$and show their representations in terms of the in-phase and quadrature spectrum of axial field $E_{z}$. These quantities are important because they represent the spectrum of the upstream half-field $E^{-}(z)$ and downstream half-field $E^{+}(z)$ defined below. Thus, $\mathcal{E}^{-}$and $\mathcal{E}^{+}$fulfil the requirements of field isolation needed by the thin-lens model.

The spectral pre-envelope $\mathcal{E}^{-}$and post-envelope $\mathcal{E}^{+}$are defined [11][12]

$$
\begin{aligned}
& \mathcal{E}^{-} \triangleq \frac{1}{2}\left(\mathcal{E}_{z}+i \mathcal{H}\left[\mathcal{E}_{z}\right]\right)=\frac{1}{2}\left(\mathcal{E}_{z}-\varepsilon_{q}\right), \\
& \mathcal{E}^{+} \triangleq \frac{1}{2}\left(\mathcal{E}_{z}-i \mathcal{H}\left[\varepsilon_{z}\right]\right)=\frac{1}{2}\left(\varepsilon_{z}+\varepsilon_{q}\right) .
\end{aligned}
$$

The pre-envelope $\mathcal{E}^{-}(i k)$ is the spectrum for the half-field

$$
\begin{aligned}
E^{-}(z) & \triangleq\left\{\begin{array}{cc}
E_{z}(z) & \text { for } z<0, \\
0 & \text { for } z>0,
\end{array}\right. \\
& =\frac{1}{2}\left[E_{z}(z)-E_{q}(z)\right],
\end{aligned}
$$

which can be shown directly by taking the Laplace transform on both sides of equality. Likewise, $\mathcal{E}^{+}(i k)$ is the spectrum for

$$
\begin{aligned}
E^{+}(z) & \triangleq\left\{\begin{array}{cc}
0 & \text { for } z<0, \\
E_{z}(z) & \text { for } z>0,
\end{array}\right. \\
& =\frac{1}{2}\left[E_{z}(z)+E_{q}(z)\right] .
\end{aligned}
$$

Thus,

$$
\begin{aligned}
& \mathcal{E}^{-}(i k)=\mathcal{L}_{2}\left[E^{-}(z)\right](i k), \\
& \mathcal{E}^{+}(i k)=\mathcal{L}_{2}\left[E^{+}(z)\right](i k) .
\end{aligned}
$$

Explicit expression for the pre- and post-envelopes in terms of the known transit-time factors are

$$
\begin{aligned}
& \mathcal{E}^{-}(i k)=\frac{V_{0}}{2}\left[T_{z}(k)-T_{q}(k)\right]-i \frac{V_{0}}{2}\left[S_{z}(k)-S_{q}(k)\right], \\
& \mathcal{E}^{+}(i k)=\frac{V_{0}}{2}\left[T_{z}(k)+T_{q}(k)\right]-i \frac{V_{0}}{2}\left[S_{z}(k)+S_{q}(k)\right],
\end{aligned}
$$

where $T_{z}, T_{q}, S_{z}, S_{z}$ are all evaluated at $k$. These are the expressions used in the numerical calculations to follow. 
The analysis also requires knowledge of the complex derivatives $\mathcal{E}^{-\prime}(\sigma)$ and $\mathcal{E}^{+^{\prime}}(\sigma)$. Since the data for $\mathcal{E}^{-}$and $\mathcal{E}^{+}$exist only on the imaginary axis $\sigma=i k$, the derivatives must be performed along this axis. Via the Cauchy-Riemann condition and analyticity [14] we get

$$
\begin{aligned}
& \left.\mathcal{E}^{-^{\prime}}(i k) \triangleq \frac{d}{d \sigma} \mathcal{E}^{-}(\sigma)\right|_{\sigma=i k}=\frac{\partial \mathcal{E}^{-}}{\partial i k}=-\frac{V_{0}}{2}\left(S_{z}^{\prime}(k)-S_{q}^{\prime}(k)\right)-i \frac{V_{0}}{2}\left(T_{z}^{\prime}(k)-T_{q}^{\prime}(k)\right), \\
& \left.\mathcal{E}^{+^{\prime}}(i k) \triangleq \frac{d}{d \sigma} \mathcal{E}^{+}(\sigma)\right|_{\sigma=i k}=\frac{\partial \mathcal{E}^{+}}{\partial i k}=-\frac{V_{0}}{2}\left(S_{z}^{\prime}(k)+S_{q}^{\prime}(k)\right)-i \frac{V_{0}}{2}\left(T_{z}^{\prime}(k)+T_{q}^{\prime}(k)\right) .
\end{aligned}
$$

The quantities $\mathcal{E}^{-}$and $\mathcal{E}^{{ }^{\prime}}$ determine the upstream gap dynamics while $\mathcal{E}^{+}$and $\mathcal{E}^{+^{\prime}}$ determine the downstream gap dynamics.

\subsection{SPECTRAL PROPERTIES}

Several additional properties of the transit time factors are needed in the analysis but are technical and not crucial to the results. The casual reader can skip to the next section without significant loss in continuity. These spectral properties result form practical aspects of the RF accelerating gap.

The physical nature of the gap electric field presumes that $E_{z}(\cdot)$ is bounded in some respect. A tool used to make this notion more precise is the dominating function. A dominating function for $E_{z}(\cdot)$, say $\bar{E}(\cdot)$, has value larger than the magnitude of $E_{z}(z)$ for every $z \in$ $\mathbb{R}$, more specifically,

$$
\bar{E}(z)>\left|E_{z}(z)\right| \quad \forall z \in(-\infty,+\infty) .
$$

Note by the above definition that $\bar{E}(z)$ is also a dominating function for $E_{q}(z)$. A gap field $E_{z}(\cdot)$

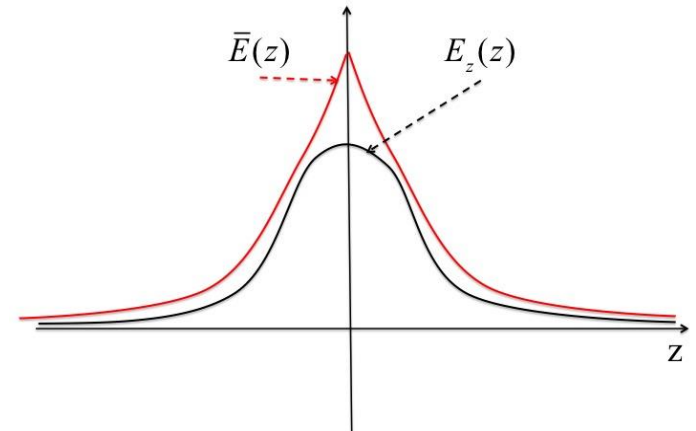

Figure 3: example dominating function originating from a real-world application invariably admits a dominating function. Let us choose the special form

$$
\bar{E}(z) \triangleq\left\{\begin{array}{lll}
M e^{+\alpha z} & \text { for } & z \in(-\infty, 0), \\
M e^{-\alpha z} & \text { for } & z \in[0,+\infty)
\end{array}\right.
$$

where $M, \alpha>0$ are real numbers. (Such an $\bar{E}(\cdot)$ helps simplify integration on the complex plane and the Laplace transform operation.) The existence of $\bar{E}(\cdot)$ comes down to the ability to find appropriate $M$ and $\alpha$ that represent the physical gap. It is reasonable to assume such numbers exist for all practical accelerating gap structures since the field is designed to be localized around $z=0$, for example, see Figure 3.

First consider the following fact concerning real integrals:

Theorem: If a function $f: \mathbb{R} \rightarrow \mathbb{R}$ is integrable and $\int_{-\infty}^{+\infty}|f(s)| d s$ exists, then

$$
\begin{aligned}
& \lim _{k \rightarrow \infty} \int_{-\infty}^{+\infty} f(s) \sin k s d s=0, \\
& \lim _{k \rightarrow \infty} \int_{-\infty}^{+\infty} f(s) \cos k s d s=0 .
\end{aligned}
$$


The idea is that as $k$ continues to increase $\sin k s$ will sample $f$ equally with positive and negative values. A proof of this theorem can be found in LePage [8].

The following spectral properties are now evident:

1) The transit time factors all have limits of zero as wave number $k$ approaches infinity in either direction. By the assumption of dominating function $\bar{E}(\cdot)$ for the axial field we have $\int_{-\infty}^{+\infty}\left|E_{z}(z)\right| d z=\int_{-\infty}^{+\infty}\left|E_{q}(z)\right| d z<$ $\int_{-\infty}^{+\infty} \bar{E}(z) d z=2 M / \alpha$ which exist for all $\alpha>0$. Thus, from their definitions and the above theorem

$$
T_{z}(k), S_{z}(k), T_{q}(k), S_{q}(k) \underset{k \rightarrow \pm \infty}{\longrightarrow} 0 .
$$

Thus by relations (33) the spectra $\mathcal{E}_{z}(\sigma), \mathcal{E}_{q}(\sigma), \mathcal{E}^{-}(\sigma)$ and $\mathcal{E}^{+}(\sigma)$ tend to zero on the imaginary axis, or

$$
\mathcal{E}_{z}(i k), \mathcal{E}_{q}(i k), \mathcal{E}^{-}(i k), \mathcal{E}^{+}(i k) \underset{k \rightarrow \pm \infty}{\longrightarrow} 0
$$

2) The transit time factors have derivatives, which have limits that also tend to zero. We show this for $T_{z}^{\prime}(k)$, the rest are analogous. Following from the definition of $T_{z}(k)$, by the smoothness of the integrand differentiate under the integral

$$
\begin{aligned}
\left|T_{z}^{\prime}(k)\right| & =\frac{1}{V_{0}}\left|\int_{-\infty}^{+\infty} z E_{z}(z) \sin k z d z\right|, \\
& <\frac{2 M}{V_{0}}\left|\int_{0}^{+\infty} z e^{-\alpha z} \sin k z d z\right| \\
& <\frac{4 M \alpha}{V_{0}}\left|\frac{k}{\left(\alpha^{2}+k^{2}\right)^{2}}\right|
\end{aligned}
$$

This gives existence for $k \neq \pm i \alpha$ (this is related to the existence of the Laplace transform). If $E_{z}(z)$ has a dominating function of the form $(36)$ then $z E_{z}(0, z)$ also has a dominating function of that form. Thus we get $T_{z}^{\prime}(k) \underset{k \rightarrow \pm \infty}{\longrightarrow} 0$. Continuing from the above

$$
\int_{-\infty}^{+\infty}\left|T_{z}^{\prime}(k)\right| d k<\frac{8 M \alpha}{V_{0}} \int_{0}^{+\infty} \frac{k}{\left(\alpha^{2}+k^{2}\right)^{2}} d k=\frac{4 M}{\alpha V_{0}}
$$

Thus, so long as there exists a finite decay $\alpha$ in the field $E_{z}(z)$ as $z \rightarrow \pm \infty$, the above theorem is applicable and

$$
T_{z}^{\prime}(k), S_{z}^{\prime}(k), T_{q}^{\prime}(k), S_{q}^{\prime}(k) \underset{k \rightarrow \pm \infty}{\longrightarrow} 0
$$

Thus, by relations (34) the spectral derivatives $\mathcal{E}_{z}^{\prime}(\sigma), \mathcal{E}_{q}^{\prime}(\sigma), \mathcal{E}^{-^{\prime}}(\sigma)$ and $\mathcal{E}^{+^{\prime}}(\sigma)$ tend to zero on the imaginary axis,

$$
\mathcal{E}_{z}^{\prime}(i k), \mathcal{E}_{q}^{\prime}(i k), \mathcal{E}^{-^{\prime}}(i k), \mathcal{E}^{+^{\prime}}(i k) \underset{k \rightarrow \pm \infty}{\longrightarrow} 0
$$


3) Transit time factors $T_{z}, S_{z}, T_{q}, S_{q}$, and their derivatives, have analytic continuations onto the complex plane; they are entire functions. However, they may have countable singularities including the point at infinity. This is essentially a statement of existence for $\mathcal{E}_{z}(\sigma)$ and $\mathcal{E}_{q}(\sigma)$ and, hence, $\mathcal{E}^{-}(\sigma)$ and $\mathcal{E}^{+}(\sigma)$. To see this return to the definition (5) of the two-sided Laplace transform. The transform is defined so long as the integral exists and the dominating function $\bar{E}(z)$ can be used to confirm existence. For example, consider the Laplace integral $L_{2}[\bar{E}]$ of the dominating function in Def. (36)

$$
L_{2}[\bar{E}](\sigma)=M \int_{-\infty}^{0} e^{(\alpha-\mu) z} e^{-i k z} d z+M \int_{0}^{+\infty} e^{-(\alpha+\mu) z} e^{-i k z} d z
$$

The first integral converges for $\operatorname{Re} \sigma<+\alpha$ and the second integral converges for $\operatorname{Re} \sigma>-\alpha$. Thus, $L_{2}[\bar{E}]$ converges in the vertical strip $-\alpha<\operatorname{Re} \sigma<+\alpha$ confirming the existence of $\mathcal{L}_{2}\left[E_{z}\right]$ on the entire complex plane via analytic continuation (except, possibly, for a countable number of singular points). Or, from a more rigorous standpoint, $T_{z}$ and $S_{z}$ represent the boundary of an analytic function $\mathcal{L}_{2}\left[E_{z}\right](\sigma)$ on the complex plane. By continuity then $T_{z}^{\prime}$ and $S_{z}^{\prime}$ are defined on the complex plane (again, except possibly for a countable number of singular points). Since $\bar{E}(z)$ is also a dominating function for $E_{q}(z) \triangleq \operatorname{sgn}(z) E_{z}(z)$ the Laplace transform $\mathcal{L}_{2}\left[E_{q}\right]$ also exists and everything previous concluded concerning $T_{z}$ and $S_{z}$ also applies to $T_{q}$ and $S_{q}$.

\section{DYNAMICS}

To determine the gap dynamics twelve parameters are required: field magnitude $V_{0}$, RF frequency $\omega$, the initial phase intercept $\phi_{0}^{-}$, the initial energy $W^{-}$, the four transit-time factors (i.e., the spectral quantities $T_{z}, S_{z}, T_{q}, S_{q}$ ) and their derivatives. The dynamics calculations are presented in four steps: 1) computing the energy gain, 2) computing the phase gain, 3) accounting for gap position offsets, and 4) formulating the system in a Hamiltonian framework.

\subsection{ENERGY GAIN}

In the thin-lens model the particle energy $W$ is constant with value $W^{-}$for all $z<0$, constant with value $W^{+}$for all $z>0$, and discontinuous with step size $\Delta W$ at $z=0$ (see Figure 2). The energy gained $\Delta W(z)$ by a particle up to axis location $z$ is given by the work done by the axial electric field $\mathfrak{E}_{z}(\cdot)$ up to position $z$ [3]. Using the harmonic expansion (2) for electric field and integrating produces

$$
\Delta W(z)=q \int_{-\infty}^{z} E_{z}(s) \cos \phi(s) d s .
$$

where $q$ is particle charge and $\phi(z)$ is the RF phase when the particle is at position $z$. Pivoting off the initial energy $W^{-}$for $z<0$ and final energy $W^{+}$for $z>0$ the above law yields the following expressions for particle energy $W(z)$ at any point in the gap region:

$$
W(z)=\left\{\begin{array}{l}
W^{-}+\int_{-\infty}^{z} q E_{z} \cos \left(\bar{k}^{-} s+\phi_{0}\right) d s \quad z<0 \\
W^{+}-\int_{z}^{+\infty} q E_{z} \cos \left(\bar{k}^{+} s+\phi_{0}\right) d s \quad z>0
\end{array}\right.
$$

where the averaged wave number approximation (4) for particle phase has been substituted for $\phi(z)$, and the $\bar{k}^{ \pm}$are a suitable average wave numbers in the upstream and downstream regions . 
Denote by $\Delta W^{-}$the definition

$$
\begin{aligned}
\Delta W^{-} & \triangleq \lim _{\substack{z \rightarrow-\infty \\
z \rightarrow}} W(0)-W(z) \\
& =W_{0}-W^{-},
\end{aligned}
$$

of the (upstream) energy gain for $z<0$ and denote by $\Delta W^{+}$the definition

$$
\begin{aligned}
\Delta W^{+} & \triangleq \lim _{z \rightarrow+\infty} W(z)-W(0) \\
& =W^{+}-W_{0}
\end{aligned}
$$

of the (downstream) energy gain for $z>0$ where $W_{0} \triangleq W(0)$ is the mid-gap energy. From the Eq. (45)

$$
\begin{aligned}
\Delta W^{-}\left(\phi_{0}, \bar{k}^{-}\right) & =q \int_{-\infty}^{0} E_{z}(s) \cos \left(\bar{k}^{-} s+\phi_{0}\right) d s \\
& =\operatorname{Re} q e^{-i \phi_{0}} \mathcal{E}^{-}\left(i \bar{k}^{-}\right), \\
\Delta W^{+}\left(\phi_{0}, \bar{k}^{+}\right) & =q \int_{0}^{+\infty} E_{z}(s) \cos \left(\bar{k}^{+} s+\phi_{0}\right) d s \\
& =\operatorname{Re} q e^{-i \phi_{0} \mathcal{E}^{+}\left(i \bar{k}^{+}\right),}
\end{aligned}
$$

where $\bar{k}^{-}$and $\bar{k}^{+}$are the upstream and downstream average wave numbers, respectively, and the Euler expansion for the exponent is used for the cosine in the integrand. The total energy gain $\Delta W$ for the gap is then equal to $\Delta W^{-}+\Delta W^{+}$.

From the continuity of $W(\cdot)$ and Eq. (45) a boundary condition can be formulated to accompany Eqs. (48). Since energy $W$ is continuous at $z=0$ both expressions in (45) are equal; that is $W_{0}=W\left(0^{-}\right)=$ $W\left(0^{+}\right)$. After recognizing $\Delta W^{-}$and $\Delta W^{+}$by the definitions

$$
\Delta W\left(k^{-}, k^{+}\right)=\Delta W^{-}\left(\phi_{0}, \bar{k}^{-}\right)+\Delta W^{+}\left(\phi_{0}, \bar{k}^{+}\right)
$$

where

$$
\Delta W\left(k^{-}, k^{+}\right) \triangleq W^{+}-W^{-},
$$

is the total energy gain of the gap. The resulting continuity equation simply states that the total energy gain from the gap is the sum of the gains from the upstream side and the downstream side. Potentially the above can be used to eliminate an additional free parameter.

\subsection{PHASE JUMP}

Computation of the phase jump $\Delta \phi$ is more involved. Consider the thin-lens piecewise linear approximation for particle phase $\phi(z)$, shown in Figure 4. Denote this approximation $\tilde{\phi}(z)$ and recognize that it asymptotically approaches $\phi(z)$ in the limits $z \rightarrow \pm \infty$. It

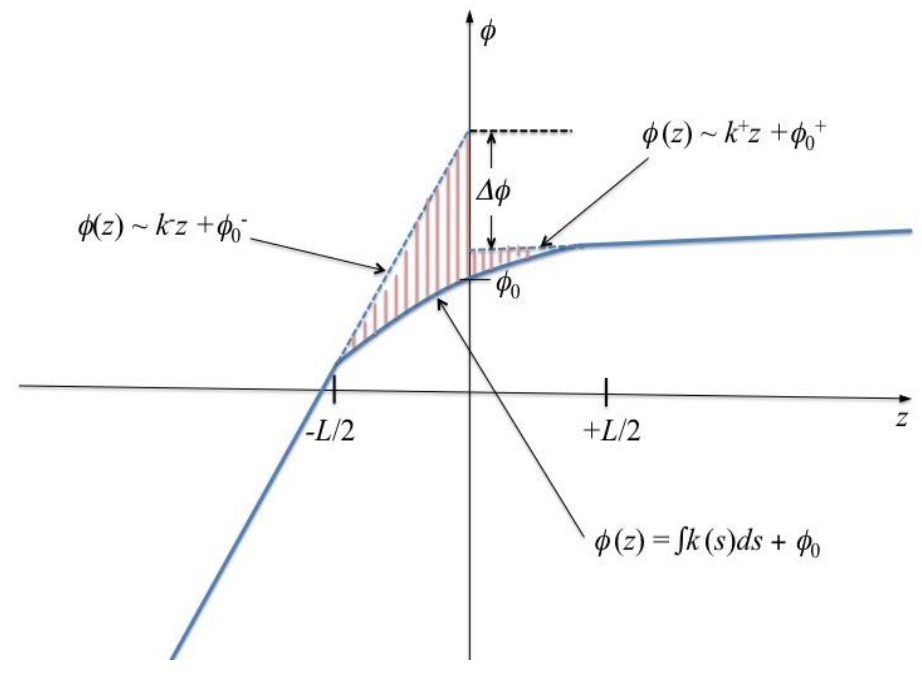

Figure 4: thin lens treatment of particle phase 
is given by

$$
\tilde{\phi}(z) \triangleq \begin{cases}k^{-} z+\phi_{0}^{-} & \text {for } \quad z<0 \\ k^{+} z+\phi_{0}^{+} & \text {for } \quad z>0\end{cases}
$$

where $k^{-}$and $k^{+}$are the (constant) pre- and post-gap wave numbers and $\phi_{0}^{-}$and $\phi_{0}^{+}$are the (constant) pre- and post-gap phase values at $z=0$. By asymptotic equivalence $\tilde{\phi}(z) \rightarrow \phi(z)$ as $z \rightarrow \pm \infty$ we have

$$
\begin{aligned}
& k^{-} \triangleq \lim _{z \rightarrow-\infty} \phi^{\prime}(z)=\lim _{z \rightarrow-\infty} k(z), \\
& k^{+} \triangleq \lim _{z \rightarrow+\infty} \phi^{\prime}(z)=\lim _{z \rightarrow+\infty} k(z),
\end{aligned}
$$

The piecewise linear approximation $\tilde{\phi}$ is discontinuous on either side of $z=0$, specifically by the intercepts

$$
\begin{aligned}
& \phi_{0}^{-} \triangleq \lim _{z \succ 0^{-}} \tilde{\phi}(z), \\
& \phi_{0}^{+} \triangleq \lim _{z \succ 0^{+}} \tilde{\phi}(z) .
\end{aligned}
$$

The desired phase jump $\Delta \phi$ is given by this discontinuity,

$$
\Delta \phi \triangleq \phi_{0}^{+}-\phi_{0}^{-} \text {. }
$$

The phase jump $\Delta \phi$ is the thin-lens mechanism needed to realign the asymptotic expressions in Eqs. (51) on either side of $z=0$. The following definitions are needed in the forthcoming analysis:

$$
\begin{aligned}
& \Delta \phi_{0}^{-} \triangleq \phi_{0}-\phi_{0}^{-}, \\
& \Delta \phi_{0}^{+} \triangleq \phi_{0}^{+}-\phi_{0},
\end{aligned}
$$

where, recall, $\phi_{0}=\phi(0)$ by construction. With the above definitions we have that $\Delta \phi=\Delta \phi_{0}^{+}+\Delta \phi_{0}^{-}$, that is, we pivot through $\phi_{0}$ in order to compute $\Delta \phi$.

To compute the phase jump we apply a variational technique expanding the wave number $k$ about the asymptotic values $k^{-}$and $k^{+}$. By differentiating wave number $k$ with respect to energy $W$ then varying with the energy gain $\Delta W(z)$ from Eq. (48) a first variation of $k$ with axis position $z$ is produced. Consider first the upstream region $z<0$. Wave number $k$ can be expressed in terms of kinetic energy $W$ as

$$
k(W)=k_{R F} \frac{W+m c^{2}}{\left(W^{2}+2 m c^{2} W\right)^{1 / 2}},
$$

where $m$ is particle mass, $c$ is the speed of light, and $k_{R F} \triangleq 2 \pi / \lambda$ is the RF wave number in free space. Expanding wave number $k$ about the initial energy $W^{-}$produces

$$
k(z) \approx k\left(W^{-}\right)+\frac{d k\left(W^{-}\right)}{d W}\left[W(z)-W^{-}\right] .
$$

Now set

$$
\begin{aligned}
& k^{-} \triangleq k\left(W^{-}\right), \\
& K^{-} \triangleq-\frac{d k\left(W^{-}\right)}{d W}=k_{R F} \frac{1}{m c^{2}} \frac{1}{\left(\beta^{-} \gamma^{-}\right)^{3}},
\end{aligned}
$$

where $\beta^{-}$is the pre-gap normalized velocity, and $\gamma^{-}$is the pre-gap relativistic factor. Factor $K^{-}$is defined so that it is a positive quantity. Substituting Eq. (45) into Eq. (57) yields the variational expression for wave number $k(z)$,

$$
k(z) \approx k^{-}-K^{-} \int_{-\infty}^{z} q E_{z}(s) \cos \left(\bar{k} s+\phi_{0}\right) d s .
$$


Now substitute this expression for $k(z)$ into Eq. (4) for upstream phase $\phi(z)$ producing

$$
\phi(z) \approx k^{-} z+\phi_{0}-K^{-} \int_{0}^{z} \int_{-\infty}^{s_{1}} q E_{z}\left(s_{2}\right) \cos \left(\bar{k}^{-} s_{2}+\phi_{0}\right) d s_{2} d s_{1},
$$

valid for $z<0$. The asymptotic nature of thin-lens phase $\tilde{\phi}$ requires $\phi(z)-\tilde{\phi}(z) \rightarrow 0$ as $z \rightarrow-\infty$. Using approximation (60) for $\phi$ and the definition (51) of $\tilde{\phi}$

$$
\begin{aligned}
0 & =\lim _{z \rightarrow-\infty}[\phi(z)-\tilde{\phi}(z)], \\
& \approx\left(\phi_{0}-\phi_{0}^{-}\right)-K^{-} \int_{0}^{-\infty} \int_{-\infty}^{s_{1}} q E_{z}\left(s_{2}\right) \cos \left(\bar{k}^{-} s_{2}+\phi_{0}\right) d s_{2} d s_{1} .
\end{aligned}
$$

Inverting the integration limits for $s_{1}$ and identifying $\Delta \phi_{0}^{-}=\phi_{0}-\phi_{0}^{-}$,

$$
\begin{aligned}
\Delta \phi_{0}^{-} & \approx-K^{-} \int_{-\infty}^{0} \int_{-\infty}^{s_{1}} q E_{z}\left(s_{2}\right) \cos \left(\bar{k} s_{2}+\phi_{0}\right) d s_{2} d s_{1} \\
& =-K^{-} \int_{-\infty}^{0} \int_{-\infty}^{z} q E_{z}(s) \cos \left(\bar{k} s+\phi_{0}\right) d s d z
\end{aligned}
$$

where $s$ and $z$ have been substituted for $s_{2}$ and $s_{1}$ as the variables of integration in the second line. The domain of integration $(s, z)$ is shown in Figure 5 for the region $s<0$. Changing the integration order in the above yields the domain $z: s<z<0, s:-\infty<s<0$, with the new integral expression

$$
\Delta \phi_{0}^{-} \approx-K^{-} \int_{-\infty}^{0} \int_{s}^{0} q E_{z}(s) \cos \left(\bar{k} s+\phi_{0}\right) d z d s .
$$

Carrying out the first integration over $z$

$$
\begin{aligned}
\Delta \phi_{0}^{-} & \approx q K^{-} \int_{-\infty}^{0} s E_{z}(s) \cos \left(\bar{k}^{-} s+\phi_{0}\right) d s, \\
& =\operatorname{Re} q K^{-} e^{-i \phi_{0}} \int_{-\infty}^{+\infty} s E^{-}(s) e^{-i \bar{k}^{-} s} d s,
\end{aligned}
$$

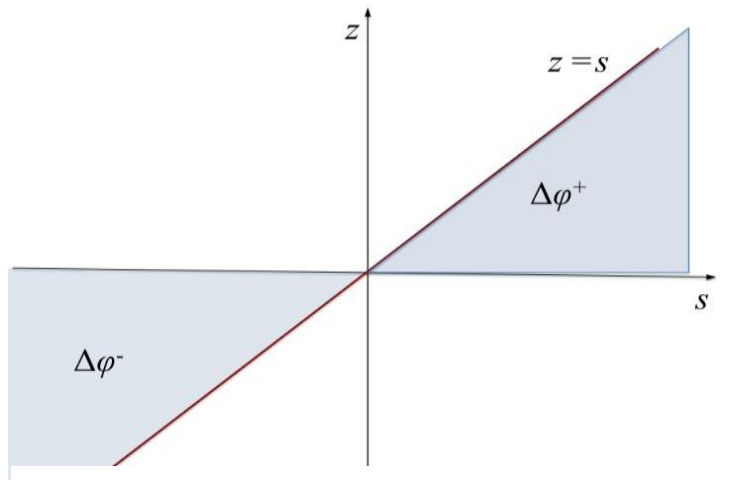

Figure 5: integration region for $\Delta \phi_{0}^{-}$and $\Delta \phi_{0}^{+}$

where the half-field $E^{-}$is substituted for $E_{z}$ in order to extend the limits of integration to the entire real line. The above integral is rearranged as

$$
\begin{aligned}
\Delta \phi_{0}^{-} & \approx \operatorname{Re} q K^{-} e^{-i \phi_{0}} \frac{-d}{d i \bar{k}^{-}} \int_{-\infty}^{+\infty} E^{-}(z) e^{-i \bar{k}^{-} z} d z \\
& =\operatorname{Re} q K^{-} e^{-i \phi_{0}}\left\{-\mathcal{E}^{-\prime}(\sigma)\right\}_{\sigma=i \bar{k}^{-}} .
\end{aligned}
$$

As seen above, the integral is recognized as the complex derivative $-d \mathcal{E}^{-}(\sigma) / d \sigma$ of the pre-envelope when evaluated at $\sigma=i \bar{k}^{-}[8]$. 
For the post-gap phase jump $\Delta \phi_{0}^{+}$start with the wave number variation analogous to Eq. (57) for $z>0$

$$
\begin{aligned}
k(z) & \approx k\left(W^{+}\right)+\frac{d k\left(W^{+}\right)}{d W}\left[W(z)-W^{+}\right], \\
& =k^{+}+K^{+} \int_{z}^{+\infty} q E_{z} \cos \left(\bar{k}^{+} s+\phi_{0}\right) d s,
\end{aligned}
$$

where (45) was substituted for $W(z)$ in the second line. Analogous definitions for the post-gap wave number $k^{+}$and derivative $K^{+}$are make as follows:

$$
\begin{aligned}
& k^{+} \triangleq k\left(W^{+}\right), \\
& K^{+} \triangleq-\frac{d k\left(W^{+}\right)}{d W}=k_{R F} \frac{1}{m c^{2}} \frac{1}{\beta^{+^{3} \gamma^{+^{3}}}},
\end{aligned}
$$

where $\beta^{+}$is the post-gap normalized velocity, and $\gamma^{+}$is the post-gap relativistic factor. Note that $K^{+}>$ 0 by definition. Proceeding as in Eq. (61) for the case $z>0$

$$
\begin{aligned}
0 & =\lim _{z \rightarrow+\infty} \phi(z)-\tilde{\phi}(z), \\
& \approx\left(\phi_{0}-\phi_{0}^{+}\right)+K^{+} \int_{0}^{+\infty} \int_{z}^{+\infty} q E_{z} \cos \left(\bar{k}^{+} s+\phi_{0}\right) d s d z .
\end{aligned}
$$

Change the order of integration so that the integration region is described $z: 0<z<s$ and $s$ : $0<s<+$ $\infty$ (see Figure 5 for the half-plane $s>0$ ). Identifying $\Delta \phi_{0}^{+}$and proceeding as before

$$
\begin{aligned}
\Delta \phi_{0}^{+} & \approx K^{+} \int_{0}^{+\infty} \int_{0}^{s} q E_{z}(s) \cos \left(\bar{k}^{+} s+\phi_{0}\right) d z d s \\
& =q K^{+} \int_{0}^{+\infty} s E_{z}(s) \cos \left(\bar{k}^{+} s+\phi_{0}\right) d s \\
& =\operatorname{Re} q K^{+} e^{-i \phi_{0}} \int_{-\infty}^{+\infty} s E^{+}(s) e^{-i \bar{k}^{+} s} d s \\
& =\operatorname{Re} q K^{+} e^{-i \phi_{0}}\left\{-\mathcal{E}^{+^{\prime}}(\sigma)\right\}_{\sigma=i \bar{k}^{+}}
\end{aligned}
$$

Collecting the results for both sides the of gap

$$
\begin{aligned}
& \Delta \phi_{0}^{-}\left(\phi_{0}, \bar{k}^{-}\right)=R e-q K^{-} e^{-i \phi_{0} \mathcal{E}^{-\prime}\left(i \bar{k}^{-}\right),} \\
& \Delta \phi_{0}^{+}\left(\phi_{0}, \bar{k}^{+}\right)=R e-q K^{+} e^{-i \phi_{0} \mathcal{E}^{+^{\prime}}\left(i \bar{k}^{+}\right) .}
\end{aligned}
$$

Eqs. (48) and (70) form the dynamics of the gap. They are coupled through the energy-momentum (i.e., wavenumber) relation (56), and definitions (58) and (67).

\subsection{GAP OFFSETS}

By the shifting property of the Laplace transform any offset $\Delta z_{0}$ of the gap electrical center (where the transit-time factors were computed) from the axis origin (where the gap is being modelled) can be represented as a multiplication of the spectra $\mathcal{E}_{z}(i k)$ and $\mathcal{E}_{q}(i k)$ by $e^{-i k \Delta z_{0}}$ [8]. Hence, the same is true for spectral quantities $\mathcal{E}^{-}(i k), \mathcal{E}^{+}(i k)$; and is true for the Hamiltonians seen in the next subsection. To see this assume the axial field profile $E_{z}(z)$ in Eq. (2) is the translation of a basis function $E_{0}(z)$ by distance $\Delta z_{0}$, that is, 


$$
\begin{aligned}
& E_{z}(z) \triangleq E_{0}\left(z-\Delta z_{0}\right), \\
& E_{q}(z) \triangleq \operatorname{sgn}\left(z-\Delta z_{0}\right) E_{0}\left(z-\Delta z_{0}\right) .
\end{aligned}
$$

Assume we know the transit-time factors $T_{z, 0}, T_{q, 0}, S_{z, 0}, S_{q, 0}$ for profile $E_{0}$, from which we construct the spectra $\varepsilon_{z, 0}, \varepsilon_{q, 0}, \mathcal{E}_{0}^{-}, \mathcal{E}_{0}^{+}$. Then for any spectral quantity $\mathcal{E} \in\left\{\varepsilon_{z}, \mathcal{E}_{q}, \mathcal{E}^{-}, \mathcal{E}^{+}\right\}$of the offset field $E_{z}(z)=$ $E_{0}\left(z-\Delta z_{0}\right)$, the substitutions

$$
\begin{aligned}
& \mathcal{E}(i k)=\mathcal{E}_{0}(i k) e^{-i k \Delta z_{0}} \\
& \mathcal{E}^{\prime}(i k)=\mathcal{E}_{0}^{\prime}(i k) e^{-i k \Delta z_{0}}-i \Delta z_{0} \mathcal{E}_{0}(i k) e^{-i k \Delta z_{0}}
\end{aligned}
$$

where $\varepsilon_{0} \in\left\{\varepsilon_{z, 0}, \varepsilon_{q, 0}, \varepsilon_{0}^{-}, \varepsilon_{0}^{+}\right\}$will yield the correct dynamics for that offset field. Note that it is not possible to apply this procedure to a field of the form $E_{q}(z)=\operatorname{sgn}(z) E_{0}\left(z-\Delta z_{0}\right)$; the alignment of functions $\operatorname{sgn}(\cdot)$ and $E_{0}(\cdot)$ cannot be changed after the transit-time factors $T_{z, 0}, T_{q, 0}, S_{z, 0}, S_{q, 0}$ are computed.

\subsection{HAMILTONIAN DYNAMICS}

First note that for a complex function $F$ of the form $F(\phi, k)=e^{-i \phi} f(k)$ where $f: \mathbb{R} \rightarrow \mathbb{C}$,

$$
\frac{\partial F}{\partial \phi}=-i F=\operatorname{Im} F-i \operatorname{Re} F
$$

or

$$
\operatorname{Re} F=\operatorname{Im}-\frac{\partial F}{\partial \phi}
$$

This fact, along with Eqs. (48) and (70), suggest the following complex "Hamiltonians" $H^{-}, H^{+}: \mathbb{C} \rightarrow \mathbb{C}$

$$
\begin{aligned}
& H^{-}(\phi+i k) \triangleq q e^{-i \phi} \mathcal{E}^{-}(i k), \\
& H^{+}(\phi+i k) \triangleq q e^{-i \phi} \mathcal{E}^{+}(i k) .
\end{aligned}
$$

In the case of a field offset $\Delta z_{0}$, applying formulas (72) the Hamiltonian functions become

$$
\begin{aligned}
& H^{-}(\phi+i k) \triangleq q e^{-i\left(\phi+k \Delta z_{0}\right)} \mathcal{E}^{-}(i k), \\
& H^{+}(\phi+i k) \triangleq q e^{-i\left(\phi+k \Delta z_{0}\right)} \mathcal{E}^{+}(i k) .
\end{aligned}
$$

Each individual Hamiltonian is independent of position but taken as a pair they are functions of position (i.e, pre-gap $H^{-}$and post-gap $H^{+}$).

For the upstream case the dynamics equations become

$$
\begin{aligned}
& \phi_{0}=\phi_{0}^{-}+\operatorname{Im}\left[K^{-} \frac{\partial}{\partial i k} H^{-}\left(\phi_{0}+\bar{k}^{-}\right)\right], \\
& W_{0}=W^{-}-\operatorname{Im}\left[\frac{\partial}{\partial \phi} H^{-}\left(\phi_{0}+\bar{k}^{-}\right)\right],
\end{aligned}
$$

where $W_{0}=W\left(k_{0}\right)$ is the (unknown) energy at the center of the gap. For the downstream case we have

$$
\begin{aligned}
& \phi_{0}^{+}=\phi_{0}+\operatorname{Im}\left[K^{+} \frac{\partial}{\partial i k} H^{+}\left(\phi_{0}+\bar{k}^{+}\right)\right], \\
& W^{+}=W_{0}-\operatorname{Im}\left[\frac{\partial}{\partial \phi} H^{+}\left(\phi_{0}+\bar{k}^{+}\right)\right],
\end{aligned}
$$

where $W^{+}=W\left(k^{+}\right)$. The final condition is the continuity of energy across the gap center $z=0$ following from Eq. (45)

$$
W^{+}-W^{-}=\operatorname{Re}\left[H^{-}\left(\phi_{0}+\bar{k}^{-}\right)+H^{+}\left(\phi_{0}+\bar{k}^{+}\right)\right] .
$$


The above is essentially a relation between the wave numbers $\bar{k}^{-}$and $\bar{k}^{+}$. Thus, the imaginary part of the Hamiltonians contains the dynamics and the real part contains energy conservation. Note the constants $K^{-}=-d k^{-} / d W$ and $K^{+}=-d k^{+} / d W$ are required since $\phi_{0}$ and $\phi_{0}^{+}$are conjugate to $W_{0}$ and $W^{+}$, not to $\bar{k}^{-}$and $k^{+}$. This inconsistency could be avoided by redefining the Hamiltonian say, for example, as $H(\phi+i W) \triangleq q e^{-i\left(\phi+k(W) \Delta z_{0}\right)} \mathcal{E}[i k(W)]$ where $k(W)$ is given by Eq. (56), thus producing a true Hamiltonian formalism.

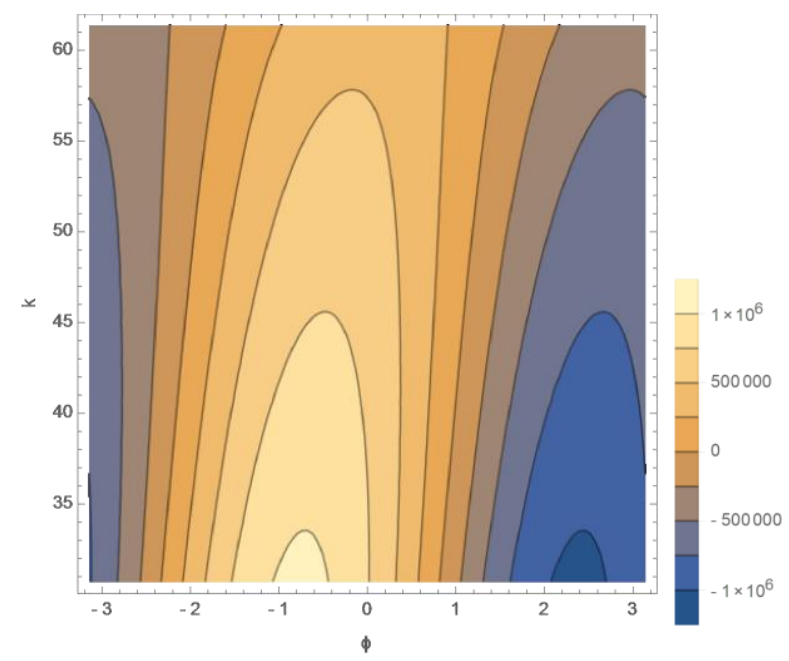

a) contour plot of $\mathrm{Im} \mathrm{H}^{-}(\phi+i k)$

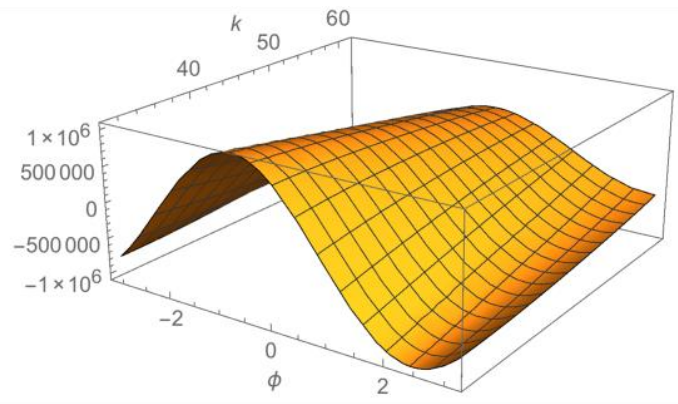

b) a) 3D plot of $I m H^{-}(\phi+i k)$

Figure 6: imaginary part of Hamiltonian function for SNS medium-beta SCL

A visualization of the Hamiltonian function $\operatorname{Im~H}^{-}(\phi+i k)$ for the entrance RF gap within the SNS medium-beta super-conducting cavity SCL Cav01a is shown in Figure 6. The first cavity in the SCL linac expects a initial beam energy $W^{-}$of about $185 \mathrm{MeV}$, which corresponds to an incident wave number of $k^{-} \approx 30.7$ radians/meter. In the figure both a contour plot and a three-dimensional plot of a pre-gap Hamiltonian are shown for $\phi \in[-\pi,+\pi)$ and a positive range of wave numbers $k$. The domain of $H^{-}$can be more precisely identified as the cylinder $[-\pi,+\pi) \times[0,+\infty)$, that is, the top half plane in $\mathbb{C}$ with edges $\operatorname{Re} \sigma=-\pi$ and $\operatorname{Re} \sigma=+\pi$ identified. Recall that particle energy $W$ scales inversely to particle wave number $k$, thus, the peaks seen in Figure 6 are actually wells with respect to energy. The reader can then visualize the accelerated "beam buckets" contained within these energy wells. In order to capture beam into these accelerating buckets, it is established practice to operate machines slightly behind the peak energy gain, usually in the region $\phi_{0} \approx-30^{\circ}$. This region is clearly visible in figure.

\section{SOLUTIONS}

Once the RF gap is identified by its spectrum, the dynamics equations have two known quantities $\phi_{0}^{-}$and $W^{-}$, five unknown quantities $\phi_{0}, \bar{k}^{-}, \phi_{0}^{+}, \bar{k}^{+}, W^{+}$, and the constituent relations (56) between energy and momentum (i.e., $k$ and $W$ ). Equations (77) and (78) provide four independent relationships between the parameters. The energy relation of Eq. (79) is not necessarily independent of the dynamics; it simply states that the total energy gain is that given by the selected $\bar{k}^{-}$and $\bar{k}^{+}$at $\phi_{0}$. Therefore, we do not have enough information to uniquely determine the gap dynamics. One must impose additional (ad hoc) assumptions to create solutions and it is wise to recognize any implications. In choosing $\bar{k}^{-}$and $\bar{k}^{+}$ note the relationship $k^{+}<\bar{k}^{+} \leq k_{0} \leq \bar{k}^{-}<k^{-}$where $k_{0}$ is the wave number at $z=0$, the center of the gap. 


\subsection{ALGORITHM 1: MID-GAP WAVE NUMBER}

We can eliminate one degree of freedom by selecting $\bar{k}^{-}$and $\bar{k}^{+}$to be the same value, specifically the mid-gap value $k_{0}$. With four unknowns $\phi_{0}, \phi_{0}^{+}, k_{0}, W^{+}$and four dynamics equations the system can be solved uniquely while ignoring the conservation equation (79). In fact, the upstream dynamics equations (77) become an independent set with unknowns $\phi_{0}, k_{0}$ ( $W_{0}$ is $k_{0}$ by another name). The dynamics equations can be iterated directly to obtain a consistent solution set when started with a value of energy less than $W_{0}$ (this fact follows from Property 1 of Subsection 2.5). Specifically, the following iterative algorithm can independently solve for the mid-gap quantities $\phi_{0}$ and $W_{0}$ given known values $\phi_{0}^{-}$and $W^{-}$ and an initial starting point $\left[\phi_{0}(0), W_{0}(0)\right]$ :

$$
\begin{aligned}
K^{-} & =K\left[W^{-}\right] ; \\
k_{0}(j) & =k\left[W_{0}(j)\right], \\
\phi_{0}(j+1) & \leftarrow \phi_{0}^{-}+\operatorname{Im} K^{-} \frac{\partial}{\partial i k} H^{-}\left[\phi_{0}(j)+i k_{0}(j)\right], \\
W_{0}(j+1) & \leftarrow W^{-}-\operatorname{Im} \frac{\partial}{\partial \phi} H^{-}\left[\phi_{0}(j)+i k_{0}(j)\right], \\
j & \leftarrow j+1 .
\end{aligned}
$$

The algorithm terminates as a Cauchy sequence. Specifically, given an error tolerance $\epsilon>0$ then algorithm (80) converges for $j>N$ if $\left|\phi_{0}(N+i)-\phi_{0}(N)\right|<\epsilon$ and $\left|W_{0}(N+i)-W_{0}(N)\right|<\epsilon$ for all $i>0$. Initializing the algorithm with the assignment $\left(\phi_{0}, W_{0}\right) \leftarrow\left(\phi_{0}^{-}, W^{-}\right)$yields acceptable convergence results (usually about 5 iterations). Once values $\phi_{0}$ and $W_{0}$ computed from the above process, the remaining unknowns are computed directly from the dynamics equations (78) as follows:

$$
\begin{aligned}
& W^{+}=W_{0}-\operatorname{Im} \frac{\partial}{\partial \phi} H^{+}\left[\phi_{0}+i k_{0}\right], \\
& k^{+}=k\left[W^{+}\right], \\
& K^{+}=K\left[W^{+}\right], \\
& \phi_{0}^{+}=\phi_{0}+\operatorname{Im} K^{+} \frac{\partial}{\partial i k} H^{+}\left[\phi_{0}+i k_{0}\right] .
\end{aligned}
$$

Thus produces $\phi_{0}^{+}$and $W^{+}$from the values $\phi_{0}$ and $W_{0}$ computed in (80) and we have the complete set.

\subsection{ALGORITHM 2: FULL PARAMETER SEARCH}

An alternate, and more complex, class of algorithms utilizes the dynamics equations and the conservation equation in order to solve for all five unknowns $\phi_{0}, \bar{k}^{-}, W_{0}, \phi_{0}^{+}, \bar{k}^{+}$, and $W^{+}$. However, here the process cannot be broken into two independent steps, all unknowns must be solved for simultaneously and self consistently. An outline for such an algorithm is described by the following:

$$
\begin{aligned}
K^{-} & =K^{-}\left[W^{-}\right] \\
K^{+}(j) & =K^{+}\left[W^{+}(j)\right] \\
k_{0}(j) & =k\left[W_{0}(j)\right] \\
k^{+}(j) & =k\left[W^{+}(j)\right] \\
\phi_{0}(j+1) & \leftarrow \phi_{0}^{-}+\operatorname{Im} K^{-} \frac{\partial}{\partial k} H^{-}\left[\phi_{0}(j), \bar{k}^{-}(j)\right] \\
W_{0}(j+1) & \leftarrow W^{-}-\operatorname{Im} \frac{\partial}{\partial \phi} H^{-}\left[\phi_{0}(j), \bar{k}^{-}(j)\right], \\
\phi_{0}^{+}(j+1) & \leftarrow \phi_{0}(j)+\operatorname{Im} K^{+}(j) \frac{\partial}{\partial k} H^{+}\left[\phi_{0}(j), \bar{k}^{+}(j)\right],
\end{aligned}
$$




$$
\begin{aligned}
W^{+}(j+1) & \leftarrow W_{0}(j)-\operatorname{Im} \frac{\partial}{\partial \phi} H^{+}\left[\phi_{0}(j), \bar{k}^{+}(j)\right], \\
\bar{k}^{-}(j+1) & \leftarrow A L G_{\bar{k}^{-}}\left[W^{-}(j+1), W^{+}(j+1), \bar{k}^{-}(j), \bar{k}^{+}(j)\right], \\
\bar{k}^{+}(j+1) & \leftarrow A L G_{\bar{k}^{+}}\left[W^{-}(j+1), W^{+}(j+1), \bar{k}^{-}(j), \bar{k}^{+}(j)\right], \\
j & \leftarrow j+1 .
\end{aligned}
$$

The implicit formulas $A L G_{\bar{k}^{-}}$and $A L G_{\bar{k}^{+}}$in the above determines the exact nature of the algorithm. They represent some "intelligent" prescription for choosing the average wave numbers $\bar{k}^{-}$and $\bar{k}^{+}$. Clearly it should be based in the conservation equation (79) and the fact that $k^{+}<\bar{k}^{+} \leq k_{0} \leq \bar{k}^{-}<k^{-}$. To date, an algorithm from this class has not been fully developed as the mid-gap wavenumber algorithm has outperformed all others tested (see Subsection 5.3). However, the most effective algorithm of this class found so far is a simple Newton-type algorithm where $A L G_{\bar{k}^{ \pm}}$uses Eq. (79) to estimate $d W / d k$ and predict the next values of $\bar{k}^{-}$and $\bar{k}^{+}$accordingly.

\subsection{VERIFICATION}

The above algorithms were implemented in software as part of a charged-particle accelerator simulation system, the details of which are described in Section 5. The operation of the software is verified by comparing the simulation results with the (numerical) solution of an equivalent analytic model consisting of a set of nonlinear, ordinary differential equations described below. If the results compare equally then the software, and hence the algorithms based upon the thin-lens model, perform as intended. Note that verification is weaker than validation, which presumes that the software does indeed accurately simulate the physical system being modelled.

The comparisons shown here are for the SNS medium-beta superconducting cavity. A field map for this cavity is shown in Figure 7. This is a six-cell, pi-mode cavity that is designed for a beta of .61, additional descriptions of the cavity can be found in [16]. The initial conditions for the beam and the cavity are taken for the entrance of the SNS superconducting linac where the initial beam energy $W^{-}$is $185.7 \mathrm{MeV}$ and we assume a synchronous phase $\phi_{0}$ of $-10^{\circ}$ at the center of the first cavity cell (modulo $\left.360^{\circ}\right)$. Note that the initial phase $\phi^{-}$is typically unknown and may have to be computed.

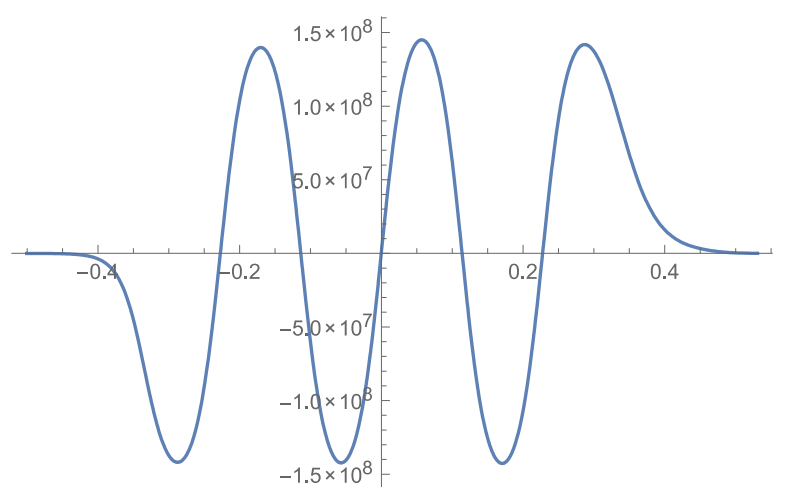

Figure 7: field map of SNS medium-beta superconducting cavity showing $E_{z}(z)$ versus axial position $z$ 
An analytic model of charged-particle acceleration is readily derived from the energy gain principle of Eq. (44) and the particle phase relation of Eq. (4). Differentiating both the equation for energy gain and the equation for phase advance with respect to axial position $\mathrm{z}$ produces the following coupled pair of ordinary differential equations:

$$
\begin{aligned}
& \phi^{\prime}(z)=k[W(z)], \\
& W^{\prime}(z)=q E_{z}(z) \cos \phi(z),
\end{aligned}
$$

where $k(W)$ is the particle wave number described by Eq. (56). Given the axial field profile $E_{z}(\cdot)$ and initial coordinates $\left(\phi^{-}, W^{-}\right)$at some initial axial position $z^{-}<0$ the above equations can be integrated numerically using standard techniques. The software package Mathematica [17] was used to generate solutions for the above equations. Typically the solutions had to be iterated in order to find the initial

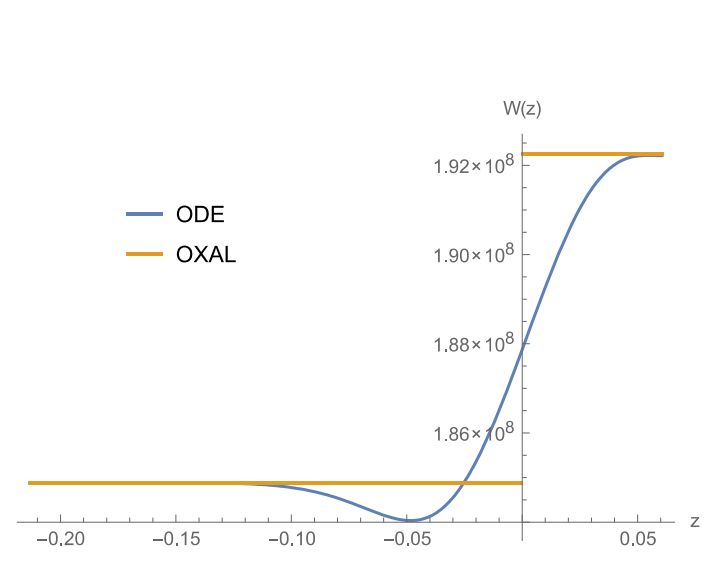

a) energy gain first cell

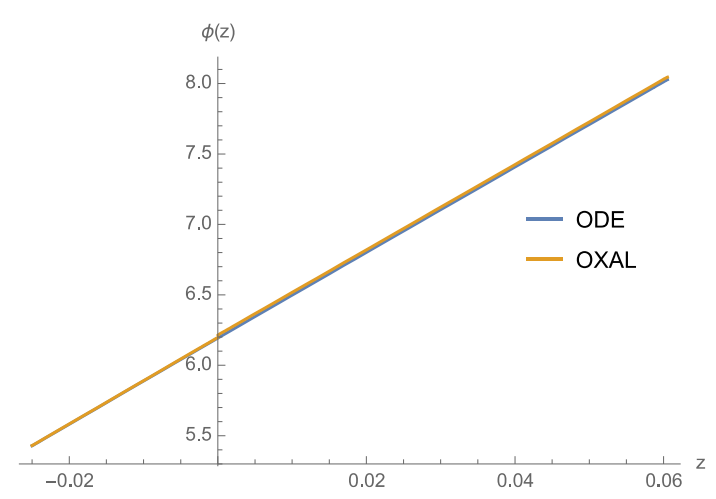

b) phase advance first cell

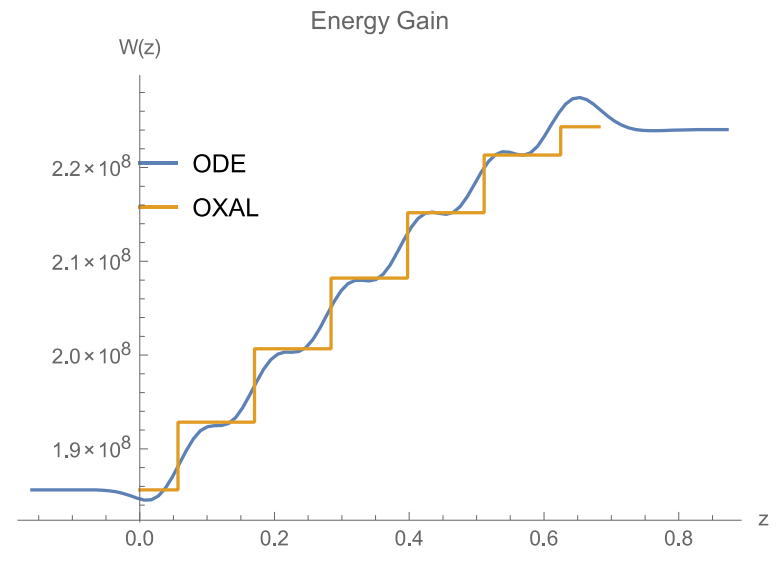

c) energy gain full cavity

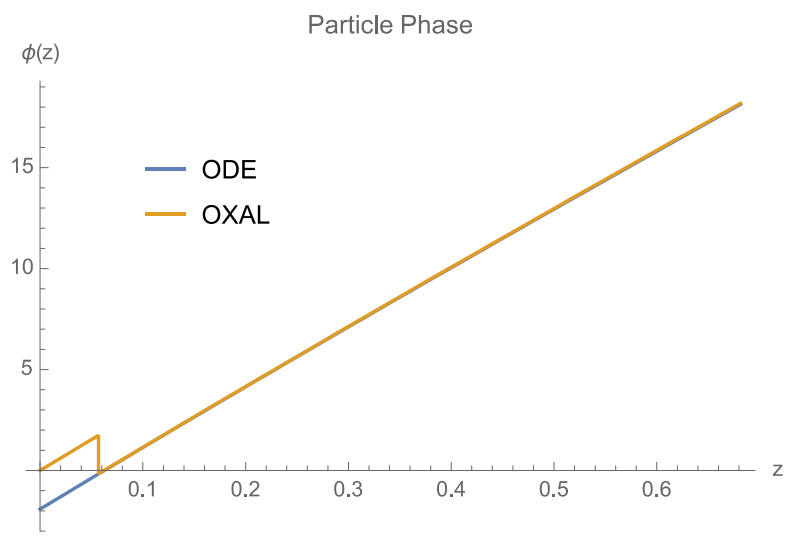

d) phase advance full cavity

Figure 8: solution comparisons for the analytic model (ODE) and thin-lens model (OXAL)

value $\phi^{-}$at $z^{-}$that produces the appropriate $\phi_{0}$ at $z=0$.

The code was successfully verified. Comparisons between the analytic model (83) and the first computer algorithm based upon Eqs. (80) and (81) are shown in Figure 8. The figure shows comparisons for a both a single RF gap, the first cell in the cavity, and the entire RF cavity. Results for the analytic model of are labeled "ODE" in the plot legends while results generated by the thin-lens computer algorithm are labeled "OXAL" (for Open XAL). When looking at the analytic results in figure a) it is clear that the particle energy initially decreases in the upstream portion of the first cell. However, this effect is "integrated out" by the thin-lens model and the appropriate energy gain is directly produced. A 
slight discrepancy in the phase advance can be seen in figure b), which is most pronounced at the gap center $z=0$. Recall the phase advance in the thin-lens model is only asymptotically accurate; accuracy improves with the distance from gap center. Referring to figure c) it is interesting to see the energy variation within the entire cavity. In particular, the particle is decelerated in the downstream portion of the last cell. This condition is due to the fact that the superconducting cavity is being operated at a particle velocity below its design value of $\beta=0.61$. The thin-lens model ignores all the detailed energy variations but yields the correct final energy. The discontinuity figure $d$ ) is due to a design artefact of the software. The software is implemented to produce a solution for a given $\phi_{0}$ at the center of the first cell; it ignores the phase advance $\phi(z)$ for all axial positions prior to that point.

\section{APPLICATION}

The results and methods described here have been implemented in several software systems. A standalone application called TTF Workshop was developed to compute the spectrum of axial fields and produce polynomial approximations that are recognized by external applications. An RF gap thin-lens acceleration model, based on Eqs. (77), (78), (79), was implemented in the Open XAL accelerator application framework according to the algorithm described in Eqs. . (80) and (81). Specifically, it was implemented within the online model software component used to simulate machine behavior. This activity was a modular addition to that of the previous algorithm based on the model described in references [1] and [2].

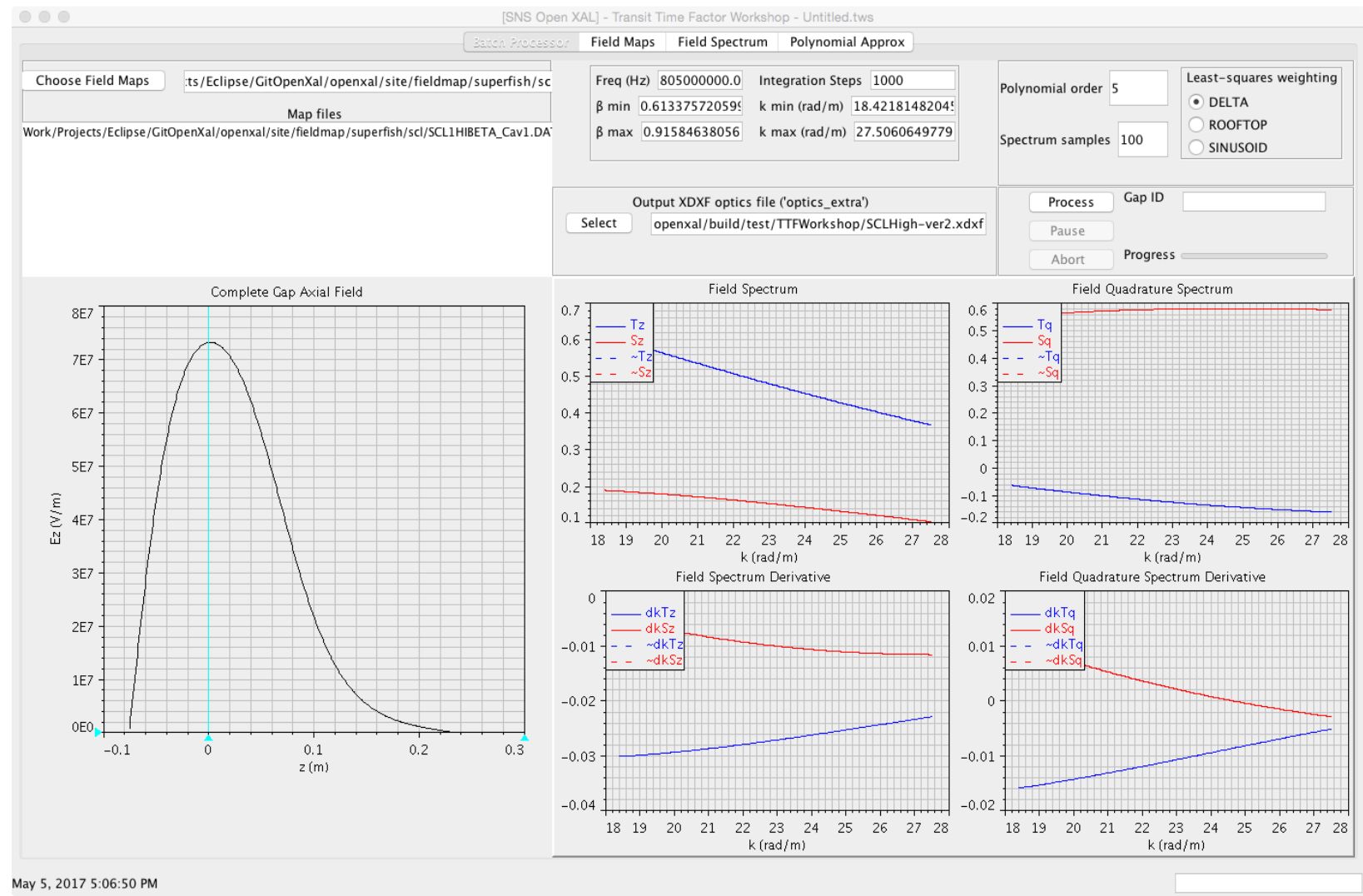

Figure 9: application TTF Workshop user interface 


\subsection{TRANSIT-TIME FACTORS}

The application TTF Workshop has been developed to generate polynomial approximations, of arbitrary degree, for all four transit-time functions $\left\{T_{z}, S_{z}, T_{q}, S_{q}\right\}$ and their derivatives. The user interface for this application is shown in Figure 9. The application takes as input superfish field map fields for the RF gaps and produces the transit-time function information in an XDXF formatted file recognized by Open XAL. (For information on the Poisson/Superfish RF cavity modelling software see the download site [18]. For information on Open XAL see the next subsection.) The application is capable of processing large file sets in batch mode, or building gap fields manually, computing the spectra, then creating polynomial approximations. A graphical user interface is available which allows the user to visualize results and fine-tune all parameters. A screenshot of TTF Workshop in batch processing mode is shown in Figure 9 where the application had finished processing the last cell of an SNS high-beta SCL cavity.

\subsection{RF GAP MODEL}

The techniques described in Section 4 have been implemented in the Open XAL online model for accelerating RF gaps. The Open XAL software system is a development environment for building highlevel control and physics applications for charged-particle accelerators. A component of the Open XAL system is the online model, which offers the application developer a machine simulator that automatically adapts and synchronizes to the current state of the accelerator hardware. For an overview of the Open XAL online model see [19]. For general information on Open XAL see the official websites and references therein [20]. The entry point of the new RF accelerating gap model is the class SpectrumMapRfGap, this class has the same interface as previous RF gap models and may be substituted using the Open XAL configuration mechanism. The hardware base classes and configuration mechanism were also modified in order to recognize the expanded spectral components $T_{z}, S_{z}, T_{q}$, and $S_{q}$.

Previously within the online model, spectral quantities were associated with cavities. That is, all gaps within cavities had the same spectral properties, with some exceptions for end cells. Along with the addition of new spectral quantities

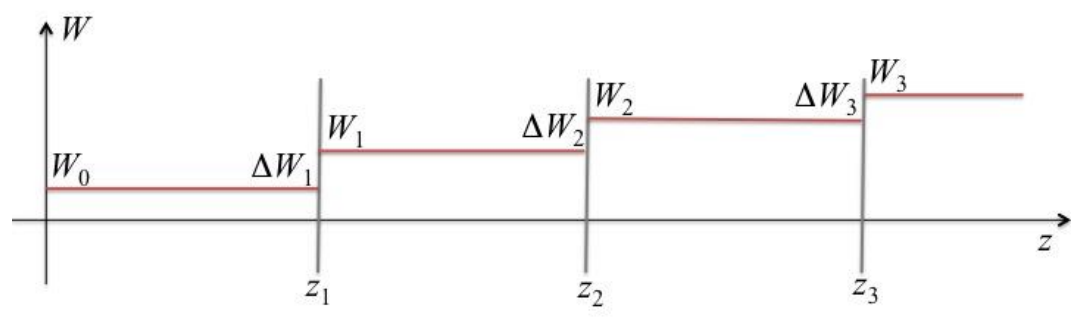
$\left(T_{q}, S_{q}\right)$, the accelerating gaps may now contain unique spectra, that is, spectral quantities are associated with individual gaps and are independent of the containing RF cavity. This is an important feature when modelling irregular cavities.

Radio frequency cavities are modelled as aggregated

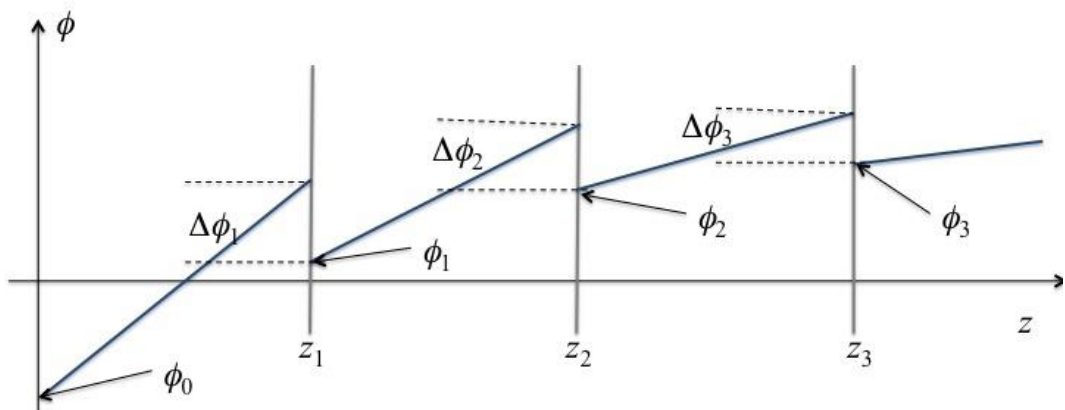

Figure 10: multi-gap phase jump and energy gain cascades of gaps; this is depicted in Figure 10. In the figure the energy is piecewise constant with jumps at the gap locations while the phase is piecewise linear with discontinuities at the gap locations. The discontinuities $\left(\Delta \phi_{n}, \Delta W_{n}\right)$ shown in the figure are the results of the phase and energy gain computations described in the paper. 


\subsection{SIMULATIONS AND PRELIMINARY COMPARISONS}

Simulation comparisons using the Open XAL online model were performed for the various accelerator sectors of the Spallation Neutron Source (SNS) linear accelerator (linac). A comparison was made between the previous acceleration model and the new acceleration model described in the paper. As mentioned earlier, the previous simulation model was implemented according to the theoretical gap model described in [1] and [2] with the addition of the odd-spectrum component $S_{z}$ described in [5]. The old simulation model does not allow independent transit-time factors for each gap and only the symmetric spectrum components were available. For the new simulation model, a variation of the mid-gap wave number algorithm of Eqs. (80) and (81) was used to solve the dynamics equations for each gap and all gaps are independently represented.

It was found that the phase jumps $\left\{\Delta \phi_{n}\right\}$ were significantly smaller than any phase advances between gaps and, thus, relatively insignificant in the overall dynamics. Maintaining accurate arrival phases $\left\{\phi_{n}\right\}$ between cells was far more important toward computing accurate values for energy gains $\left\{\Delta W_{n}\right\}$ for the new algorithm. Thus, use of the mid-gap wave number $k_{0}$ approach described in Subsection 4.1 was adequate and fast.

Presented in Figure 11 are simulation results from the Open XAL online model. With regards to the figure, two situations are of primary interest, warm structures and cold structures. Results throughout the SNS warm linac did not demonstrate any significant differences. A plot of energy computed by both old and new methods is shown in Figure 11a) for the SNS DTL1 cavity, the curves are essentially indistinguishable. More interesting findings were seen in cold structures. A comparison for the SNS medium-beta super-conducting cavity SCL Cav01a is shown in Figure 11b). There the initial acceleration falls off in the new model but then accelerates more strongly in the later portion of the structure. The final energies are nearly equal with the new model yielding a value approximately $-0.5 \%$ of the old model results. However, simulating the entire cold linac a difference of $-2.4 \%$ is observed. The final errors for the warm structures are typically of the order $0.1 \%$ or less.

It is important to note at this point that an additional source of error is the gap offsets $\Delta z_{n}$. The gap offsets used for the previous RF gap model were known and used in the simulation results. However, the gap offsets for the new model are as of yet unavailable and so were ignored in the simulation results. These offsets need to be determined for the new model and populated into the parameters database. At the time of this writing those values were unavailable and thus it is unknown if they contribute significantly to the overall results.

With respect to the above caveat there are some additional factors that can be attributed for the simulation discrepancies. Some differences between the two model results can be attributed to significant differences in the warm and cold structures of the SNS linac. Although the warm linac does have some inherit asymmetry, it is not enough to create significant effects in the dynamics, thus, the extra modelling capabilities of the new implementation is not expressed. The end cells of the warm cavities exhibit the most field irregularity, however, they still do not produce a significant odd spectral component in the transit time factors. Additionally, most of the warm cavities are regular and, thus, have identical spectral components. That is, having unique spectra for each gap in that case is unnecessary. On the other hand, the cold cavities create fields with significant asymmetries and gap irregularities; this condition is clearly seen in the field profile for the end cell shown in the TTF workshop screen shot of Figure 9. The new model is able to represent these features of the field profile and they appear in the dynamics. 
To reiterate, in the old model, all the internal RF gaps within a parent RF cavity are essentially identical. The spectral data for all gaps within the cavity are shared, there are however provisions for
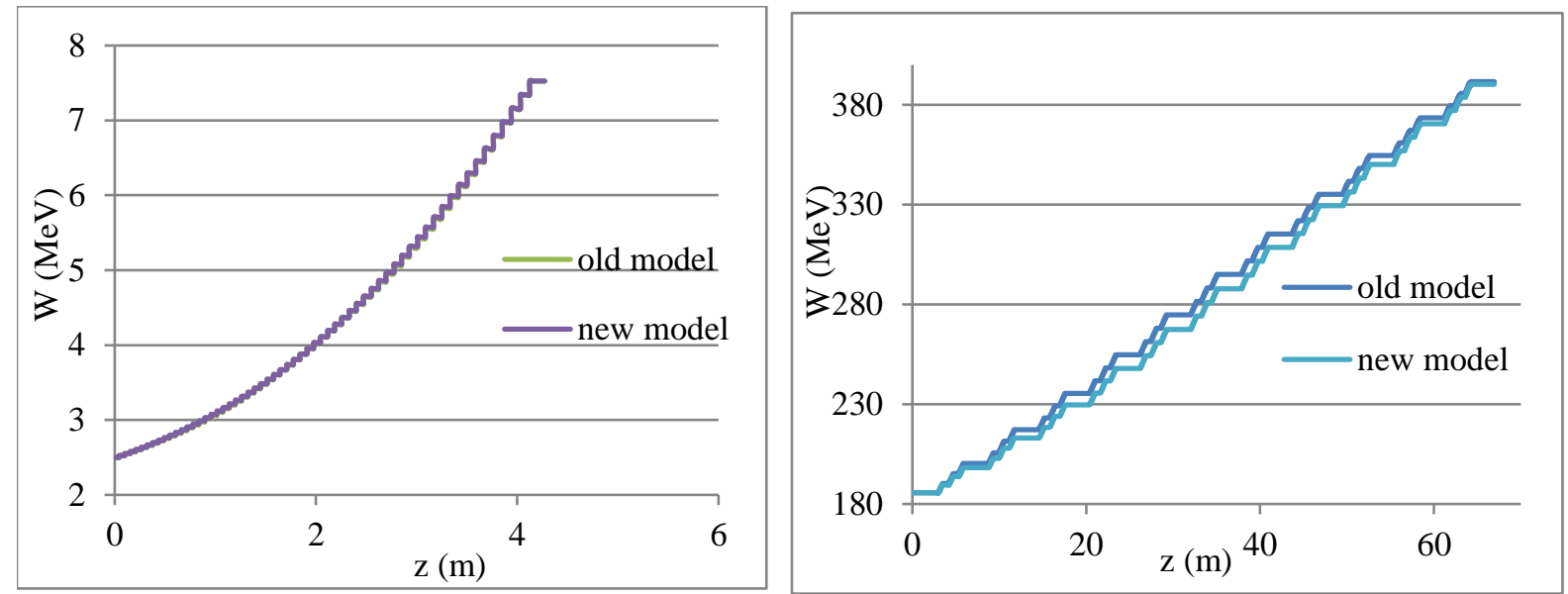

Figure 11: simulation comparison for a) SNS DTL1 and b) SNS medium-beta SCL

identifying gaps at the exit and entrance. This capability appears to be sufficient when modelling the warm linac where the fields are regular (at least for the Superfish field maps available [18]). However, the super-conducting cavities have gap fields that are more tightly coupled and non-regular. The capability to model these conditions has a significant effect in this case.

\section{CONCLUSION}

The current RF accelerating gap thin-lens model compliments previous treatments of the thin-lens gap seen in the literature in that the approach is from a systems and signal processing standpoint and yields a discrete Hamiltonian-like dynamics description. The spectral analysis provided by the Laplace and Hilbert transforms provides a theoretically cohesive framework (i.e., systems theoretic) for treatment of the thin-lens model and several standard results from system theory are used to generate the results (e.g., convolution theorem, shifting theorem, derivative theorem). An important aspect of this approach is the identification of the pre- and post-envelope spectrum that isolate the upstream and downstream axial field profile, respectively, and serve the role of generalized transit time factors. With the full spectral compliment the thin-lens gap model allows consideration of any axial electric field profile, including asymmetric fields and offset fields. This paper is an expanded form of one presented earlier at the IPAC2017 conference, it includes the formula derivations and further results.

The thin-lens RF gap model presented here was incorporated into the Open XAL online model. Each RF gap element can be given its own individual set of transit-time factors that are represented by approximating polynomials valid over a given interval of wave numbers. This situation allows the concurrent tracking of the synchronous particle trajectory along with the collective beam bunch dynamics. The transit-time factors are computed using a separate tool TTF workshop that inputs cavity field maps then creates files of polynomial coefficients and their domains in the Open XAL format. Note that it is still necessary to identify the relative "operating phase" of individual cavities; this is typically done using some type of phase scanning process as described in [4].

Simulation studies were performed to both verify the operation of the new RF gap model implementation in the Open XAL online model, and compare the operation with that of the previous acceleration model. The verification was done by comparing results from the computer simulation with that of an analytic model represented by a set of nonlinear, ordinary differential equations. Both the computer model and the analytic model produced the equivalent results thus verify the software 
operation. The SNS linac was simulated using both the previous gap model and the new RF gap model to compare the performance of each implementation. It was found that when gap geometries are irregular or vary significantly within an RF cavity the new model shows observable differences, on the order of a per cent in energy gain. This effect may be significant, for example in cold accelerating structures. However, for cavities with regular geometries the new acceleration model implementation offers little advantage over the previous implementation. 


\section{REFERENCES}

[1] P. Lapostolle, "Proton Linear Accelerators: A Theoretical and Historical Introduction," LA-11601MS, July, 1989, Chapt. 5.

[2] P. Lapostolle and M. Weiss, "Formulae and Procedures Useful for the Design of Linear Accelerators," CERN-PS-2000-001

[3] T. Wangler, Principles of RF Linear Acceleration (Wiley, New York, 1998), Sect. 2.2, 7.3.

[4] T.L. Owens, M.B. Popovic, E.S. McCrory, C.W. Schmidt, and L.J. Allen, "Phase Scan Signature Matching for Linac Tuning," Particle Accelerators Vol. 48, pp. 169-179 (1994).

[5] A. Shishlo and J. Holmes, "Physical Models for Particle Tracking Simulations in the RF Gap," ORNL TechNote ORNL/TM-2015/247 (June, 2016).

[6] C.K. Allen, "On the Computation of Phase and Energy Gain for a Thin-Lens RF Gap Using a General Field Profile,” MOPIK117, IPAC2017, Copenhagen, Denmark, May 14-19, 2017.

[7] C.K. Allen, “A Thick-Len Model for Charged-Particle RF Accelerating Gaps," ORNL Tech. Report, in progress.

[8] W.R. LePage, Complex Variables and the Laplace Transform for Engineers (Dover, New York, 1961), Chapt. 11, p. 350, Chapt. 10, p. 285, 308, 310.

[9] E. Stein and G. Weiss, Introduction to Fourier Analysis on Euclidean Spaces (Princeton University Press, 1971).

[10] E.C. Titchmarsh, Introduction to the theory of Fourier integrals (2nd ed.), (Oxford University: Clarendon Press 1948).

[11] S. Haykin, Communication Systems, Third Edition (Wiley, 1994), Appendix A6.3.

[12] S.A. Tretter, Communication System Design Using DSP Algorithms (Springer, 2015), Chapt. 5.

[13] S. Olver, "Computing the Hilbert Transform and its Inverse," Math. Comp. Vol. 80 (2011), pp. 1745-1767.

[14] J. Brown and R. Churchill, Complex Variables and Applications (McGraw-Hill, 9 Ed., 2013), Chap. 2.

[15] V.I. Arnold, Mathematical Methods of Classical Mechanics (Springer-Verlag, 2 edition, 1989). p. 65.

[16] S.H. Kim, M. Doleans, and Y. Kang, "Efficient Design Scheme of Superconducting Cavity," LINAC2000, Monterey, CA, 2000, pp. 923-925.

[17] Mathematica technical support http://www.wolfram.com/mathematica.

[18] Poisson/Superfish download site: http://laacg.lanl.gov/laacg/services/download_sf.phtml.

[19] C.K. Allen, T.A. Pelaia, J. Freed, "Architectural Improvements and New Processing Tools for the Open XAL Online Model,” IPAC2015, Richmond, Virginia, USA, May 3-8, 2015, MOPWI047.

[20] Open XAL Project Site, https://openxal.github.io and https://github.com/openxal. 